\title{
Afroneutria, a new spider genus of Afrotropical Ctenidae (Arachnida, Araneae)
}

\author{
Daniele POLOTOW ${ }^{1,{ }^{*}}$ \& Rudy JOCQUÉ ${ }^{2}$ \\ ${ }^{1}$ Instituto Butantan, Laboratório Especial de Coleções Zoológicas, Av. Vital Brasil, 1500, Butantã, São \\ Paulo, CEP 05503-000, Brazil. Corresponding author: danielepolotow@gmail.com \\ ${ }^{2}$ Royal Museum for Central Africa, Tervuren, Belgium. Email: rudy.jocque@africamuseum.be \\ ${ }^{1}$ urn:lsid:zoobank.org:author:133DA297-CBF8-4F7B-BD52-9A917F663594 \\ ${ }^{2}$ urn:lsid:zoobank.org:author:CF15016C-8CD1-4C9D-9021-44CA7DC7A5D5
}

\begin{abstract}
The new ctenid genus Afroneutria is proposed to include five species: Afroneutria velox (Blackwall, 1865) comb. nov. (type species), A. immortalis (Arts, 1912) comb. nov., A. erythrochelis (Simon, 1876) comb. nov., A. quadrimaculata sp. nov. and A. hybrida sp. nov., all collected in central to southeast Africa. Afroneutria species can be distinguished from the remaining Ctenidae by the presence of a synapomorphic laminar projection on the embolus tip. The genus can also be distinguished by the presence of the following set of characters: elongated cup-shaped median apophysis and elongated and laminar embolus on the male palp; large lateral projections on the epigynum and the spermathecae divided into two parts, dorsal and ventral, in the vulva.
\end{abstract}

Keywords. Africa, Ctenus, systematics, taxonomy

Polotow D. \& Jocqué R. 2015. Afroneutria, a new spider genus of Afrotropical Ctenidae (Arachnida, Araneae). European Journal of Taxonomy 121: 1-27. http:/dx.doi.org/10.5852/ejt.2015.121

\section{Introduction}

Ctenidae Keyserling, 1877 is composed of nocturnal and cursorial spiders, common in the lower strata of tropical forests of South and Central America, Asia and Africa. To date, the family comprises 491 species, distributed in 40 genera (World Spider Catalog 2014). Currently, most of the Ctenidae species are placed in the genus Ctenus Walckenaer, 1805. Based on a new diagnosis and recent cladistic analysis, Ctenus is considered polyphyletic as currently delimited (Silva 2003; Simó \& Brescovit 2001; Brescovit \& Simó 2007; Polotow \& Brescovit 2009, 2014).

According to the phylogenetic hypothesis proposed by Polotow \& Brescovit (2014), several Ctenus species need to be allocated to new genera, including a clade formed by Ctenus velox Blackwall, 1865, C. immortalis Arts, 1912 and C. erythrochelis Simon, 1876. They appear as a well-supported group, not related to the type species of the genus, Ctenus dubius Walckenaer, 1805 (Polotow \& Brescovit 2014: fig. 3, clade D). 
The aim of this paper is to establish the new Afrotropical ctenid genus Afroneutria to accommodate five species: Afroneutria velox (Blackwall, 1865) comb. nov. (type species), A. immortalis (Arts, 1912) comb. nov., A. erythrochelis (Simon, 1876) comb. nov., A. quadrimaculata sp. nov. and A. hybrida sp. nov., all collected in central and East Africa. We also reestablish A. immortalis comb. nov., previously considered a synonym of $A$. velox comb. nov.

\section{Material and methods}

Morphological observations and illustrations were made using a Wild M10 and a Wild M5 stereo microscope. The epigynum was detached from the abdomen and submerged in methyl salicylate to clear the internal structures. For SEM, specimens were cleaned ultrasonically, dried through critical point, gold coated, and then examined and photographed with a JEOL $6480 \mathrm{LV}$ scanning electron microscope at the Royal Museum for Central Africa, Tervuren, Belgium (MRAC). All measurements are in millimeters.

The following abbreviations are used below:

$\begin{aligned} \mathrm{ALE} & =\text { anterior lateral eyes } \\ \mathrm{AME} & =\text { anterior median eyes } \\ \mathrm{C} & =\text { conductor } \\ \mathrm{CD} & =\text { copulatory ducts } \\ \mathrm{Cy} & =\text { cymbium } \\ \mathrm{d} & =\text { dorsal } \\ \mathrm{E} & =\text { embolus } \\ \mathrm{FD} & =\text { fertilization ducts } \\ \mathrm{LL} & =\text { locking lobes } \\ \mathrm{LS} & =\text { lateral sector } \\ \mathrm{MA} & =\text { median apophysis } \\ \mathrm{MS} & =\text { median sector } \\ \mathrm{p} & =\text { prolateral } \\ \mathrm{PLE} & =\text { posterior lateral eyes } \\ \mathrm{PME} & =\text { posterior median eyes } \\ \mathrm{r} & =\text { retrolateral } \\ \mathrm{RTA} & =\text { retrolateral tibial apophysis } \\ \mathrm{S} & =\text { spermathecae } \\ \mathrm{Te} & =\text { tegulum } \\ \mathrm{Ti} & =\text { tibiae } \\ \mathrm{v} & =\text { ventral }\end{aligned}$

The material examined is deposited in the following institutions (curator in parentheses):

BMNH $=$ The Natural History Museum, London (J. Beccaloni)
MNHN $=$ Muséum National d'Histoire Naturelle, Paris (C. Rollard)
MRAC $=$ Royal Museum for Central Africa, Tervuren (R. Jocqué)
OUMNH $=$ Oxford University Museum of Natural History (Z. Simmons)
ZMB $=$ Zoological Museum of Berlin (J. Dunlop)
ZMUC




\title{
Results
}

\author{
Class Arachnida Cuvier, 1812 \\ Order Araneae Clerck, 1757 \\ Family Ctenidae Keyserling, 1877 \\ Subfamily Cteninae Keyserling, 1877 \\ Genus Afroneutria gen. nov. \\ urn:1sid:zoobank.org:act:59ECDA85-C86E-416E-A98D-CB1DD6AB5DA1
}

\section{Type species}

Ctenus velox Blackwall, 1865. Designated here.

\section{Diagnosis}

Afroneutria species can be distinguished from the remaining Ctenidae by the presence of a synapomorphic laminar projection on the embolus tip (Fig. 1B; Polotow \& Brescovit 2014: character 25, fig. 3). The genus can also be distinguished from the remaining Cteninae spiders by the presence of the following set of characters: elongated cup-shaped median apophysis and elongated and laminar embolus on the male palp (Fig. 1A-B); large lateral projections on the epigynum and the spermathecae divided into two parts, dorsal and ventral, in the vulva (Fig. 1C).

\section{Etymology}

The generic name is a combination of the first part of Afrotropical and the last part of Phoneutria Perty, 1833, a Neotropical genus that bears superficial similarities with the genus described here. The gender is feminine.

\section{Description}

Ecribellate ctenids. Total body length (males and females) 12.40-32.00. Carapace piriform (Fig. 2A, C), brown, covered by white hairs in longitudinal median area and in lateral area; tuft of long white hairs between PME; border with white hairs; thoracic groove longitudinal, in posterior third; except A. erythrochelis comb. nov., which is covered by golden hairs. Chilum divided. Clypeus with long erect black bristles. Ctenid eye pattern 2-4-2, with anterior and posterior row recurved in dorsal view. Eyes round, except oval anterior lateral eyes. Chelicerae dark brown, covered by white hairs (except A. erythrochelis comb. nov., covered with golden hairs); promargin with three teeth, the median higher than the laterals; retromargin with four similar sized teeth; prominent basal condyle; intermarginal denticles present only in A. immortalis comb. nov. and A. hybrida sp. nov. Endites reddish brown with lighter apical area, external lateral margin excavated, distally bearing dense apical scopulae and subapical serrula. Labium dark brown, distally rounded, bearing dense apical scopula. Sternum reddish brown, oval, not extending between coxae IV. Legs reddish brown. Male legs more elongated and slender than female legs. Trochanter notched. Tarsus with claw tufts (Fig. 1D-F) composed of tenent setae, specialized in adhesion to smooth surfaces (Ramírez 2014). Abdomen oval, with tufts of white hairs and numerous black setae on anterior dorsal area (Figs 2A-D, 4A-D, 6A-D, 8A-D, 10A-B). Males and females with specific ventral coloration pattern on abdomen (Figs 2B, D; 4B, D; 6B, D; 8B, D; 10B). Male palp (Figs $1 \mathrm{~A}-\mathrm{B}, 3 \mathrm{~A}-\mathrm{C}, 5 \mathrm{~A}-\mathrm{C}, 7 \mathrm{~A}-\mathrm{B}, 9 \mathrm{~A}-\mathrm{C}, 11 \mathrm{~A}-\mathrm{B}, 13 \mathrm{~A}-\mathrm{D}, 14 \mathrm{~A}-\mathrm{B}, 15 \mathrm{~A}-\mathrm{D})$ : tibia with short VTA; short RTA with three projections, except $A$. erythrochelis comb. nov. with two projections and $A$. hybrida sp. nov. with truncated tip; cymbium with elongated tip; subtegulum prolateral, except $A$. erythrochelis comb. nov. with retrolateral subtegulum; tegulum oval; median apophysis cup-shaped and elongated; embolus elongated with retrolateral area hyaline and complex tip; locking lobes present; conductor short and partially sclerotized. Epigynum (Figs 3D-E, 5D-E, 7C-D, 9D-E, 12A-D): divided in median field and two lateral fields; median field with anterior area narrow and posterior area swollen and large; lateral 

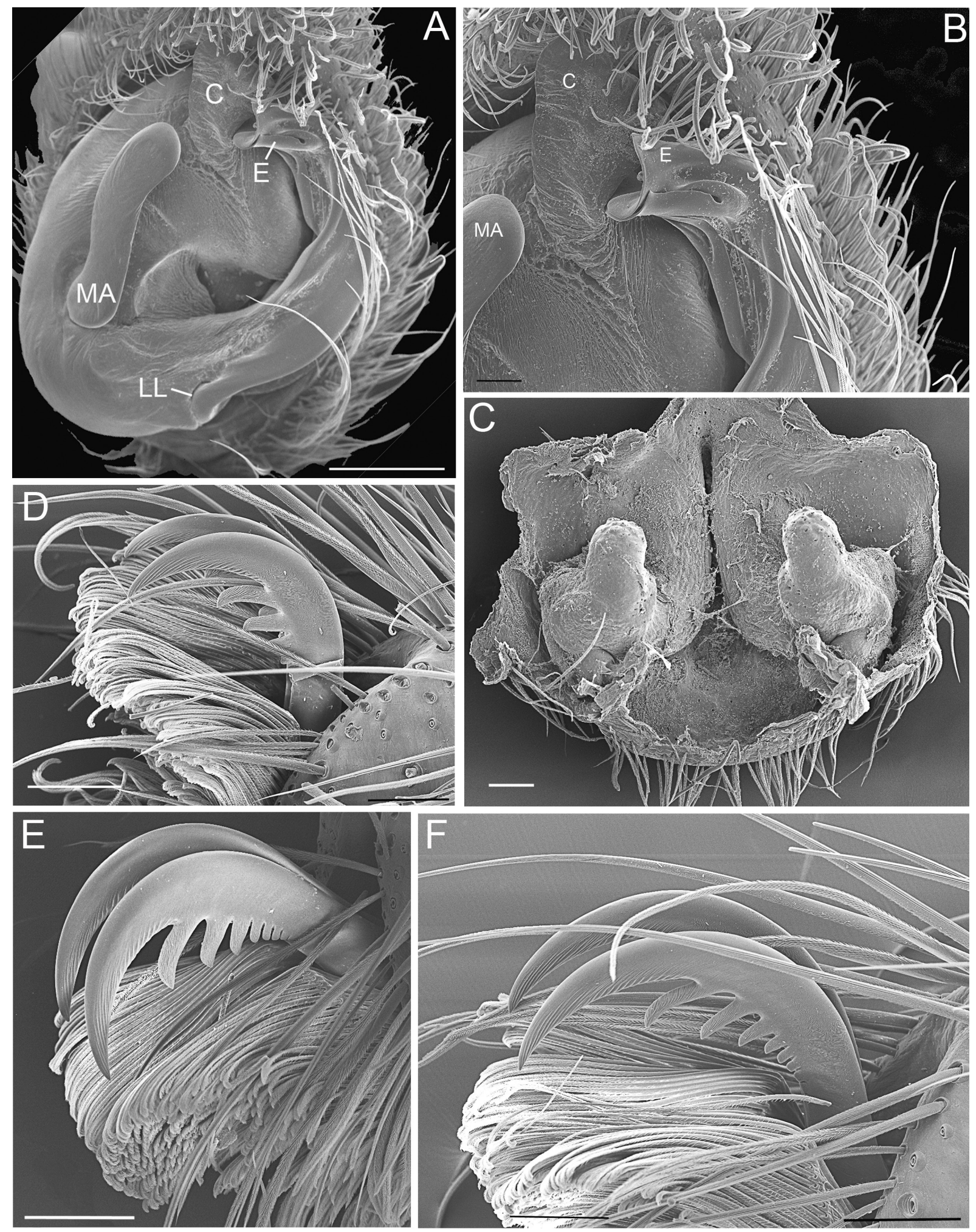

Fig. 1. Afroneutria velox (Blackwall, 1865) comb. nov.. - A-B. Male palp, ventral view. - C-F. o. C. Epigynum, dorsal view. D-F. Tarsus, lateral view. D. Leg III. E. Leg I. F. Leg IV. Scale bars = $200 \mu \mathrm{m}$. 
field with lateral projections; lateral projections covering part of median field; copulatory opening with lighter area; spermathecae divided in two parts, ventral and dorsal; copulatory duct ending in ventral part; fertilization duct arising of dorsal part (Figs 1C, 3D-E, 5D-E, 7C-D, 9D-E).

\section{Composition}

Five species: Afroneutria velox comb. nov., A. immortalis comb. nov., A. erythrochelis comb. nov., A. quadrimaculata sp. nov. and $A$. hybrida sp. nov.

\section{Distribution}

Central and southeast Africa (Figs 16A-B).

Afroneutria velox (Blackwall, 1865) comb. nov.

Figs 1A-F, 2A-D, 3A-E, 12D, 13A-B, 16A

Ctenus velox Blackwall, 1865: 336.

Phoneutria melanogastra Bösenberg \& Lenz, 1895: 36, pl. 1, fig. 14.

Ctenus marshalli F.O. Pickard-Cambridge, 1898: 26, pl. 4, figs 12-13.

Ctenus velox-F.O. Pickard-Cambridge 1898: 15, pl. 3, figs 1-2. - Benoit 1977: 700, fig. 2a-d.

Ctenus melanogaster - Strand 1907: 694. - Arts 1912: 196, pl. 1, fig. 14a-b. — Benoit 1977: 700, fig.

2a-d (synonymised with C. velox).

Ctenus melanogastra - Hyatt 1954: 884.

Ctenus marshalli - Hyatt 1954: 884 (synonymised with C. melanogaster).

\section{Note}

Blackwall (1865) indicated one female as the type material used in the description of Ctenus velox, but two adult females were found in the OUMNH collection and, according to the label, both were designated as types. Based on the original description we here designate the smaller specimen as the lectotype and the other female as the paralectotype.

\section{Diagnosis}

The males of Afroneutria velox comb. nov. differ from males of the other species of the genus by the laminar and truncated tip and the additional subdistal projection of the embolus (Fig. 1B) and the shape of the RTA (Fig. 3A-C). The females can be distinguished from the remaining species of the genus by the acuminated and short lateral projections of the epigynum and rounded dorsal spermathecae in the vulva (Fig. 3D-E).

\section{Material examined}

\section{Type material}

MOZAMBIQUE: Ctenus velox, female lectotype (designated here), 1 female paralectotype, next to confluence of Shiré River with Zambezi River (approximate coordinates: 1740’24.0” S, 35¹7'51.2” E), OUMNH 1554.

\section{Additional material}

TANZANIA: Kipatimu, 1 đૈ, 1965, T.H. Hofman coll. (MRAC 137443); Kyela, 1 †, 9 Nov. 1991, R. Jocqué coll. (MRAC 173465); 1 ㅇ, 27 Nov. 1991, R. Jocqué coll. (MRAC 173191); Coast Province,

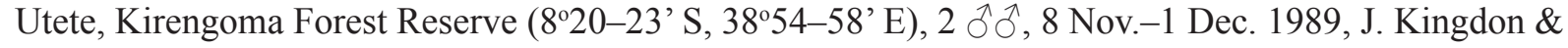
Frontier Tanzania coll. (ZMUC); Coast Province, Bagamoyo, Zaraninge (Kiono) Forest Reserve, $20 \mathrm{~km}$ SW of Sadaani (604’ S, 38³5’ E), 2 $^{\wedge}{ }^{\circ}, 1$ ㅇ, Mar. 1990, Frontier Tanzania coll. (ZMUC). 


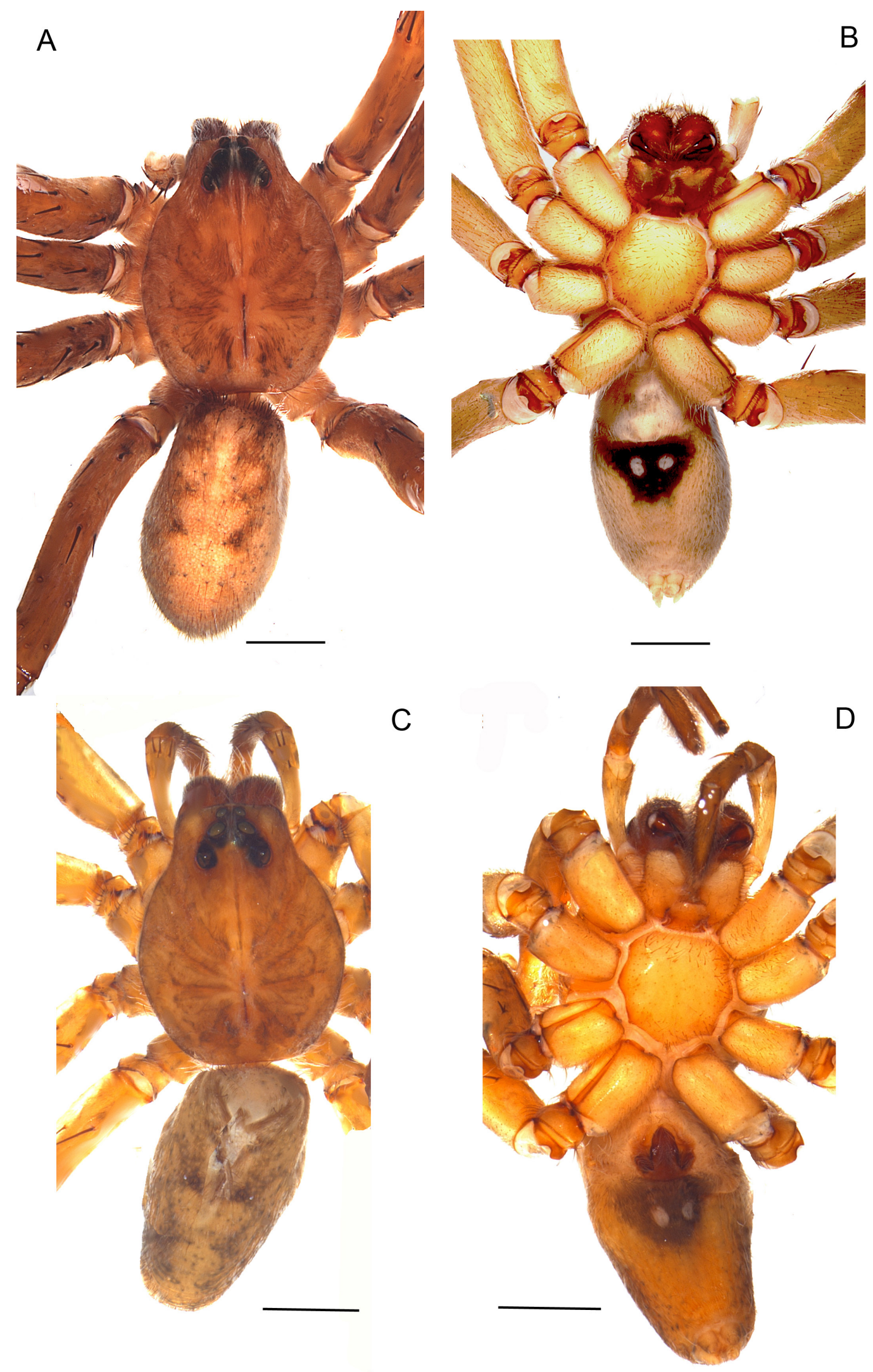

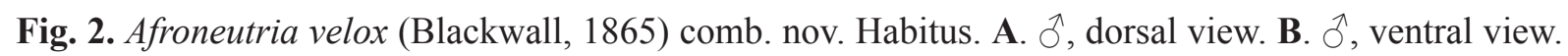
C. , , dorsal view. D. , , ventral view. Scale bars $=1 \mathrm{~mm}$. 

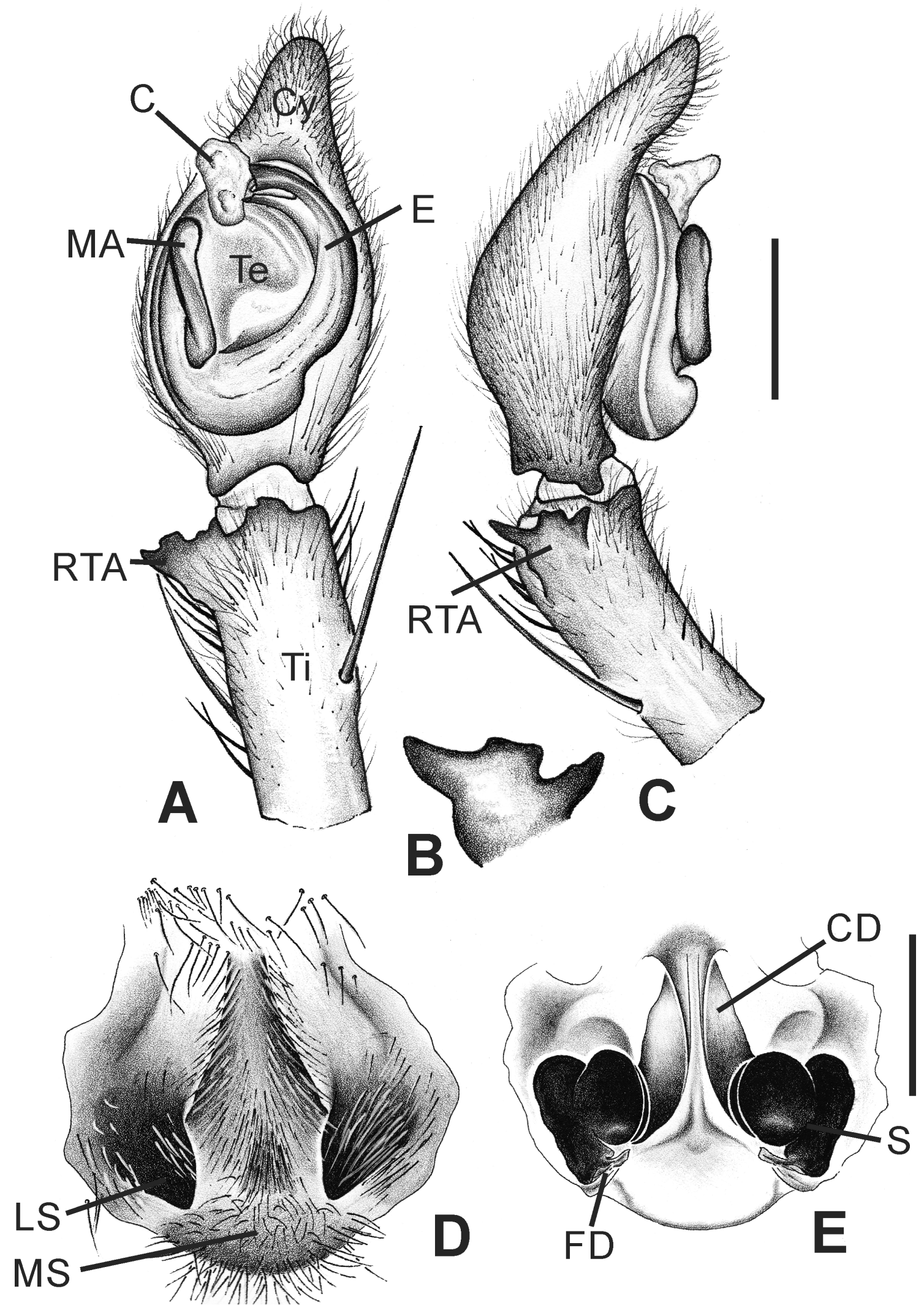

Fig. 3. Afroneutria velox (Blackwall, 1865) comb. nov. - A-C. Male palp. A. Ventral view. B. Retrolateral view. C. Retrolateral tibial apophysis, lateral view. - D-E. Female genitalia. D. Epigynum, ventral view. E. Vulva, dorsal view. Scale bars $=1 \mathrm{~mm}$. 
MALAWI: Chintheche, 1 đ̊, 1 †, 1-20 Nov. 1977, R. Jocqué coll. (MRAC 155299).

DEMOCRATIC REPUBLIC OF THE CONGO: Elisabethville, Katanga, 1 +, 30 Jan. 1931, Bredo coll. (MRAC 15545); Buttgenbach, Lubumbashi, 1 (MRAC 25506).

\section{Type material not examined}

EAST AFRICA: Phoneutria melanogastra, 2 male syntypes, 1 female syntype, deposited in Zoologisches Museum Hamburg.

ZIMBABWE: Ctenus marshalli, holotype, ô, Umfuli River, also known as Mupfure River (approximate coordinates: $17^{\circ} 55^{\prime} 13.3^{\prime \prime}$ S, 2956'40.7” E), Mashonaland, BMNH.

\section{Description}

\section{Male (MRAC 155299)}

Total length 12.30. Carapace 6.20 long and 4.60 wide. Eye diameter: AME 0.32, ALE 0.18, PME 0.60, PLE 0.56. Leg measurements: I: femur 8.40 / patella 3.20 / tibia 8.70 / metatarsus 8.60 / tarsus 3.30 / total 32.20; II: 8.10 / 3.30 / 7.40 / 7.60 / 2.80 / 29.20; III: 6.80 / 2.60 / 5.70 / 6.40 / 2.30 / 23.80; IV: 9.30 / $2.60 / 8.90 / 11.10 / 3.30 / 35.20$. Leg formula 4123. Leg spination: tibia I and II v2-2-2-2-2, r1-1, p1-1; III and IV v2-2-2, r1-1, p1-1; metatarsus I, II and III v2-2-2, p1-1-1, r1-1-1; IV v1-1-1-1-1-2, r1-1-1, p1-1-1. Coloration: venter of abdomen black, with two white spots placed transversely behind epigastric furrow (Fig. 2B). Palp: cymbium elongated, longer than tibia; conductor rectangular; median apophysis slightly curved (Figs 1A-B, 3A-C, 13A-B).

\section{Female (MRAC 173465)}

Total length 18.60. Carapace 8.10 long and 6.40 wide. Eye diameter: AME 0.36, ALE 0.24, PME 0.60, PLE 0.48. Leg measurements: I: femur 6.80 / patella 3.10 / tibia 6.30 / metatarsus 6.00 / tarsus 2.20 / total 24.40; II: 6.30 / 2.90 / 5.40 / 5.50 / 2.10 / 22.20; III: 5.20 / 2.30 / 4.50 / 5.10 / 2.10 / 19.20; IV: $7.50 / 2.60 / 6.60 / 8.40 / 2.30 / 27.40$. Leg formula 4123. Leg spination: tibia I and II v2-2-2-2-2, r0, p0; III and IV v2-2-2, r1-1, p1-1; metatarsus I and II v2-2-2, r0, p0; III v2-2-2, r1-1-1, p-1-1-1; IV v11-1-1-1-2, r1-1-1, p1-1-1. Coloration: ventral abdomen black, with two white spots placed transversely behind epigastric furrow (Fig. 2C-D). Epigynum: triangular median field and short lateral projections of lateral fields of epigynum (Fig. 3D). Dorsal spermathecae short and round; ventral spermathecae with rebordered lateral margins (Figs 1C, 3E, 12D).

\section{Variation}

Two ふす: total length 10.60-12.40, carapace length 6.00-6.30, femur I length 7.10-8.70. Three 우: total length $16.40-18.70$, carapace length 7.00-8.10, femur I length 6.20-6.50. At least two $\widehat{\partial} \widehat{\partial}$ present venter of abdomen black in front, red towards back, with two spots placed transversely behind epigastric furrow.

\section{Distribution}

Tanzania, Malawi, Mozambique, Democratic Republic of the Congo and Zimbabwe (Fig. 16A).

Afroneutria immortalis (Arts, 1912) revalidated, comb. nov. Figs 4A-D, 5A-E, 12A, 14A-B, 16A

Ctenus immortalis Arts, 1912: 202, pl. 2, fig. 25.

Ctenus velox-Benoit 1977: 700, fig. 2a-d (misidentified and wrongly synonymised with C. immortalis). Ctenus immortalis - Polotow \& Brescovit 2014: 335, 338, 348, 355. 


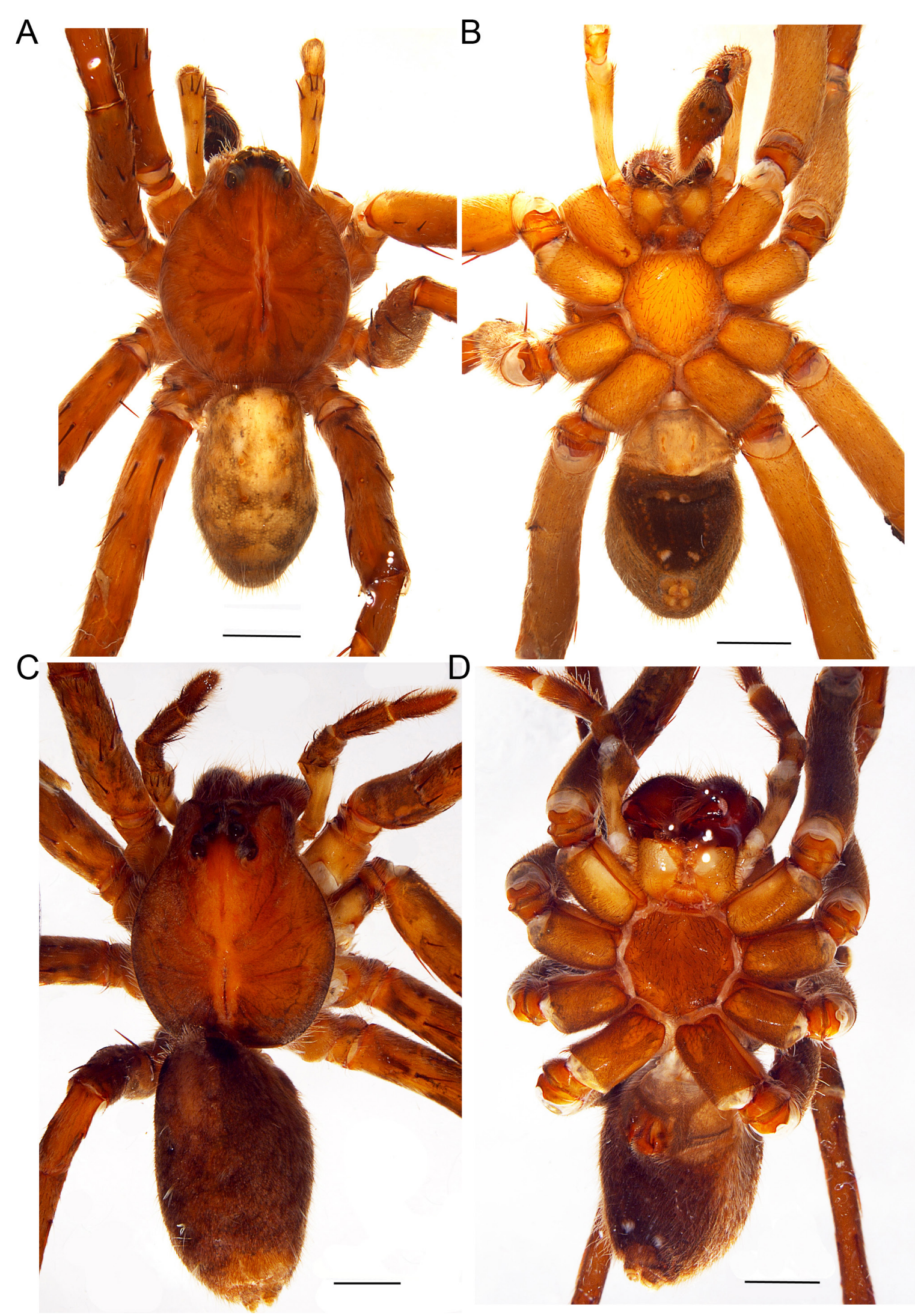

Fig. 4. Afroneutria immortalis (Arts, 1912) comb. nov. Habitus. A. §̊, dorsal view. B. §, ventral view.

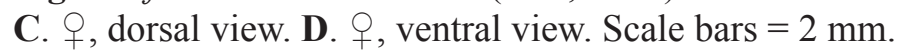



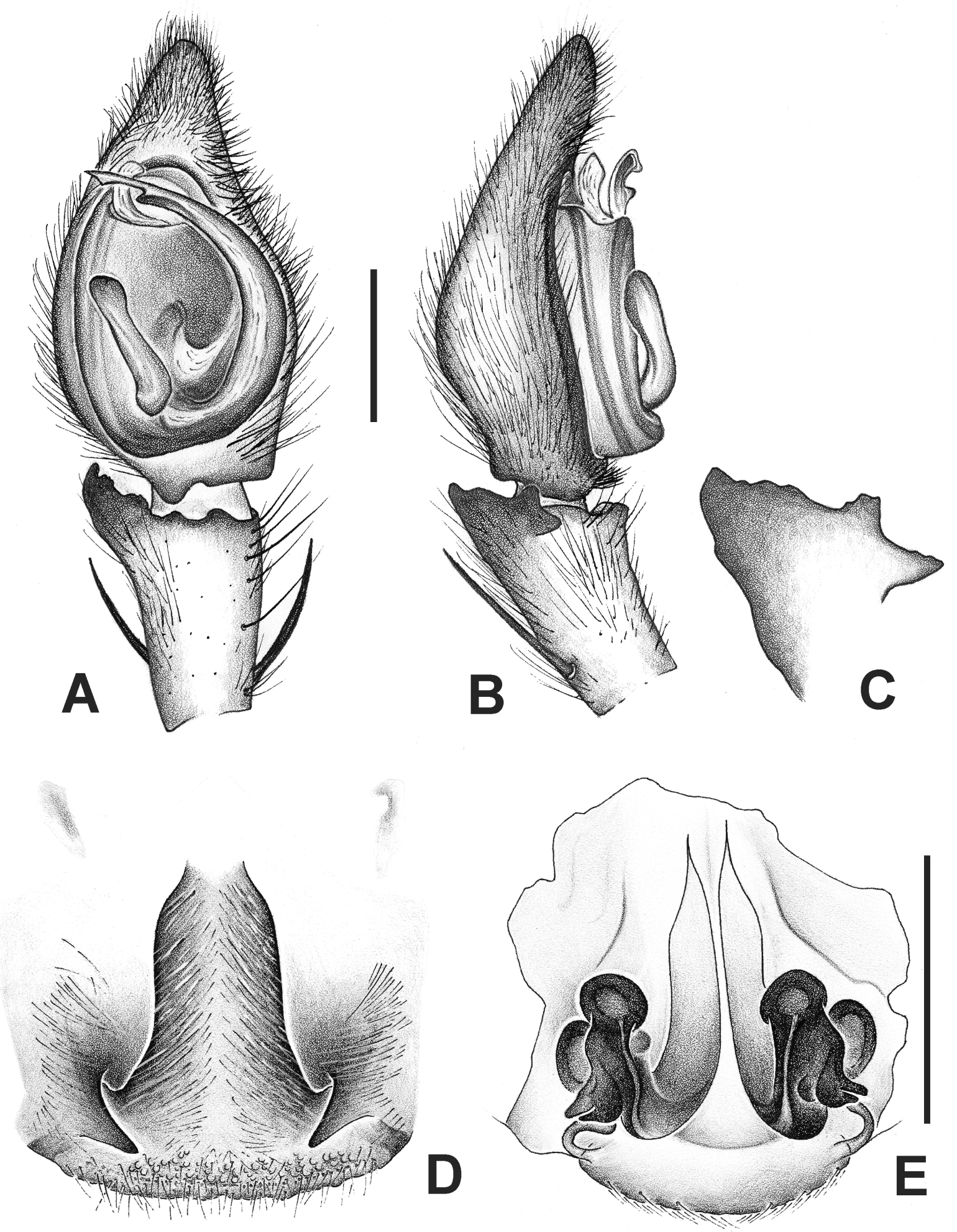

Fig. 5. Afroneutria immortalis (Arts, 1912) comb. nov. - A-C. Male palp. A. Ventral view. B. Retrolateral view. C. Retrolateral tibial apophysis, lateral view. - D-E. Female genitalia. D. Epigynum, ventral view. E. Vulva, dorsal view. Scale bars $=1 \mathrm{~mm}$. 


\section{Diagnosis}

The males of Afroneutria immortalis comb. nov. differ from those of the other species of the genus by the elongated tip and short additional laminar projection of the embolus on the male palp (Figs 5A, 14A; Polotow \& Brescovit 2014: fig. 8f, indicated by an arrow) and the shape of the RTA (Fig. 5C; Polotow \& Brescovit 2014: fig. 8d). The females can be distinguished by the acute lateral spurs (Figs 5D, 12A) and straight dorsal and ventral spermathecae with a swollen apical area (Fig. 5E).

\section{Material examined}

\section{Type material}

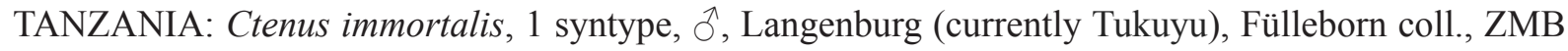

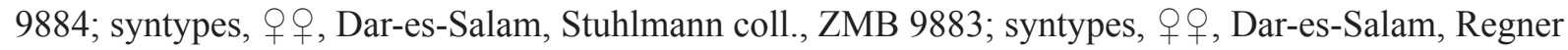
coll., ZMB 9881.

KENYA: Ctenus immortalis, syntypes, 1 , 2 immature, Kibwezi, Schütter coll., ZMB 9882 and 9885.

\section{Additional material}

KENYA: Coast Province, Arabuko Sokoke National Park, 1 q, 25 Sep. 2001, D. Van den Spiegel coll. (MRAC 211539); Tsavo, Taita West National Park, Taita Hills, Mwachora, pitfalls, 1 đ, 20 Jun. 1999, D. Van den Spiegel coll. (MRAC 208828); Tsavo, Taita Discovery Center, 1 đ̄, 27 Mar. 2000, R. Jocqué \& C. Warui coll. (MRAC 209543); Watamu, 1 §, 1977, J.L. Cloudsley-Thompson coll. (MRAC 148774).

TANZANIA: Amani, Amani Forest Reserve, 1 q, Dec. 1978, K.M. Howell coll. (MRAC 159481); Mkomazi Game Reserve, Ibaya Hill, 1 ð, 24 Nov. 1994, A. Russell-Smith coll. (MRAC 213883); Coast Province, Kisarawe, Kasimzumbwi Forest Reserve, 6057' S, 3903' E, 1 ㅇ, Jan.-Feb. 1991, Frontier Tanzania coll. (ZMUC); Coast Province, Mafia Island, 753' S, 3950’ E, 2 우 응. Oct.-Nov. 1990, Frontier Tanzania coll. (ZMUC).

\section{Description}

Male (MRAC 213883)

Total length 13.40. Carapace 7.20 long and 5.60 wide. Eye diameter: AME 0.32, ALE 0.18, PME 0.60, PLE 0.56. Leg measurements: I: femur 8.60 / patella 3.40 / tibia 8.90 / metatarsus 8.90 / tarsus 3.40 / total 33.20; II: 8.10 / 3.30 / 7.40 / 7.60 / 2.80 / 29.20; III: 6.80 / 2.60 / 5.70 / 6.40 / 2.30 / 23.80; IV: 9.50 / $2.80 / 9.10 / 11.30 / 3.50 / 36.20$. Leg formula 4123. Leg spination: tibia I and II v2-2-2-2-2, r1-1, p1-1; III and IV v2-2-2, r1-1, p1-1; metatarsus I, II and III v2-2-2, p1-1-1, r1-1-1; IV v1-1-1-1-1-2, r1-1-1, p1-1-1. Coloration: venter of abdomen black, with two white spots in front of spinnerets (Fig. 4B). Palp: cymbium elongated, longer than tibia; conductor triangular; median apophysis slightly curved, with acute tip (Figs 5A-C, 14A-B).

\section{Female (MRAC 211539)}

Total length 16.40. Carapace 7.00 long and 5.40 wide. Eye diameter: AME 0.36, ALE 0.24, PME 0.60, PLE 0.48. Leg measurements: I: femur 6.80 / patella 3.00 / tibia 6.40 / metatarsus 6.00 / tarsus 2.20 / total 24.40; II: 6.30 / 2.90 / 5.40 / 5.50 / 2.10 / 22.20; III: 5.20 / 2.40 / 4.40 / 5.10 / 2.00 / 19.10; IV: 7.50 / $2.60 / 6.60 / 8.40 / 2.30 / 27.40$. Leg formula 4123. Leg spination: tibia I and II v2-2-2-2-2, r0, p0; III and IV v2-2-2, r1-1, p1-1; metatarsus I and II v2-2-2, r0, p0; III v2-2-2, r1-1-1, p-1-1-1; IV v1-1-1-1-12, r1-1-1, p1-1-1. Coloration: venter of abdomen black, with two white spots in front of spinnerets (Fig. 4D). Epigynum: triangular median field and elongated lateral projections of lateral field (Figs 5D, 12A). Dorsal and ventral spermathecae elongated (Fig. 5E). 


\section{Variation}

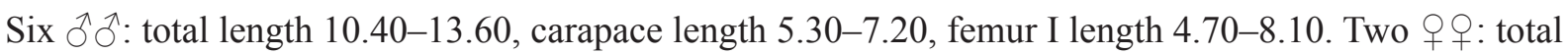
length 15.70-16.40, carapace length 7.00-7.60, femur I length 6.80-7.90. Several $q$ + $q$ with four white spots on venter of abdomen, two behind epigastric furrow, two in front of spinnerets.

\section{Distribution}

Kenya and Tanzania (Fig. 16A).

Afroneutria erythrochelis (Simon, 1876) comb. nov.

Figs $6 \mathrm{~A}-\mathrm{D}, 7 \mathrm{~A}-\mathrm{D}, 15 \mathrm{C}-\mathrm{D}, 16 \mathrm{~B}$

Phoneutria erythrochelis Simon, 1876: 222.

Phoneutria auricularis Karsch, 1879: 347.

Ctenus johnstoni F.O. Pickard Cambridge, 1898: 21, pl. 3, fig. 7.

Ctenus carsoni F.O. Pickard Cambridge, 1898: 24, pl. 3, figs 4-5.

Ctenus aureopubescens Strand, 1906: 82.

Ctenus erythrochelis - Simon 1897: 107, figs 98, 101. — Lessert 1915: 44, pl. 2, fig. 33.

Phoneutria auricularis - Arts 1912: 188, pl. 1, fig. 1.

Ctenus johnstoni - Arts 1912: 188, pl. 1, fig. 1.

Ctenus carsoni - Arts 1912: 188, pl. 1, fig. 1.

Ctenus aureopubescens - Benoit 1977: 698, figs 1a-d.

\section{Diagnosis}

The males of Afroneutria erythrochelis comb. nov. differ from those of the other species of the genus by the swollen retrolateral area of the cymbium, the curved median apophysis, the retrolateral base of the embolus and by the laminar projection of the embolus extending beyond its tip (Figs 7A-B, 15CD). The females can be distinguished by the short lateral projections of the epigynum and the dorsal spermathecae curved ventrally, forming a spiral in the vulva (Figs 7C-D, 12C).

\section{Material examined}

Type material

ANGOLA: Phoneutria erythrochelis, holotype, $\widehat{\partial}$, Landana, MNHN.

ANGOLA: Phoneutria auricularis, syntypes, 2 ถึ, 2 $+q, 2$ immature, Benguela, Chinchoxo, Falkenstein coll., ZMB 2563.

\section{Additional material}

ANGOLA: Distrito do Lunda, Cuango, $9^{\circ} 10^{\prime} \mathrm{S}, 17^{\circ} 58^{\prime} \mathrm{E}, 1$ ôn, Sep. 1972, C. Cabral coll. (MRAC 167914).

BURUNDI: Bujumbura, 323' S, 2921' E, 1 , 1959, G. Marlier coll. (MRAC 114120); Kanyania,

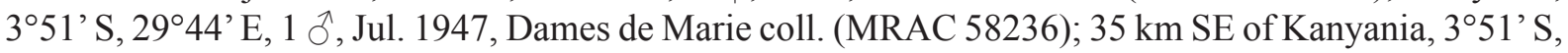
2944’ E, 1 đ’, 1948, Dames de Marie coll. (MRAC 60880).

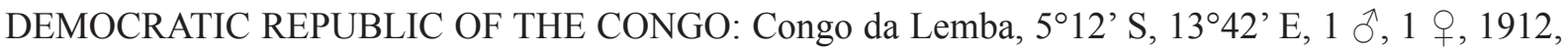
R. Mayné coll. (MRAC 16303); Ekoli, terr. Opola, $0^{\circ} 22^{\prime}$ S, $2^{\circ}{ }^{\circ} 16^{\prime}$ E, 2 q 9 , 1952 , Meews coll. (MRAC 73889, 73890); Funda Biabo, 950' S, 2533' E, 1 ô, 2 Apr. 1914, L. Charliers coll. (MRAC 25490); Katako-Kombe, $3^{\circ} 22^{\prime}$ S, $24^{\circ} 24^{\prime}$ E, 1 क , 10 Apr. 1952, Dr. Fontaine coll. (MRAC 72460); Chutes Guillaume, Kwango, 7²4' S, $17^{\circ} 18^{\prime}$ E, 1 ò, 1936, G. Schmitz coll. (MRAC 21546); Lomami, 9²4' S,

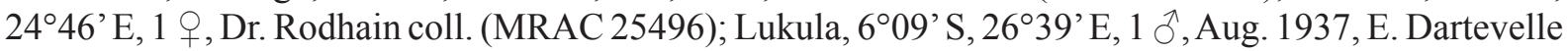



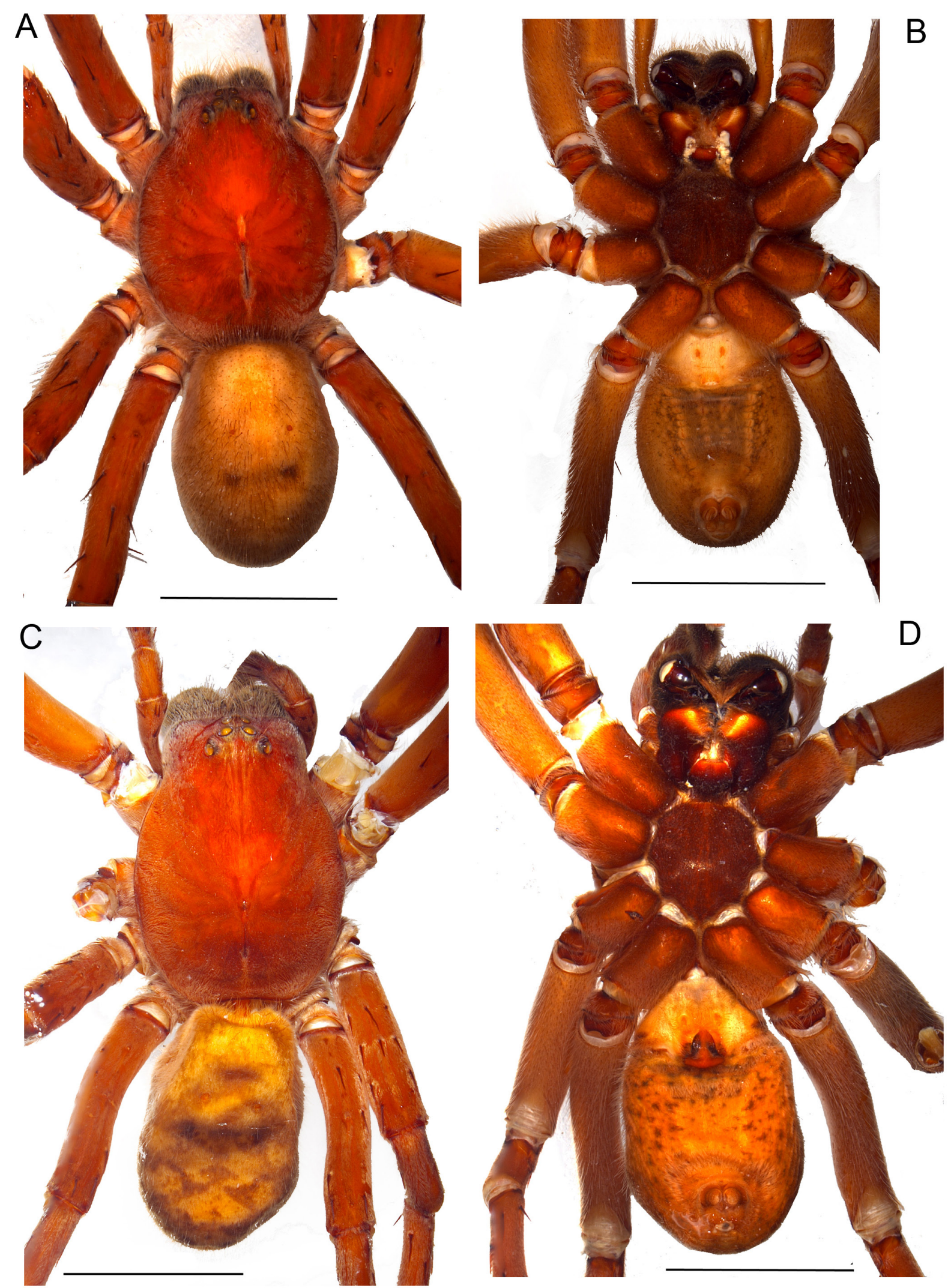

Fig. 6. Afroneutria erythrochelis (Simon, 1876) comb. nov. Habitus. A. $\widehat{\partial}$, dorsal view. B. $\widehat{\partial}$, ventral view. C. , dorsal view. D. + , ventral view. Scale bars $=10 \mathrm{~mm}$. 

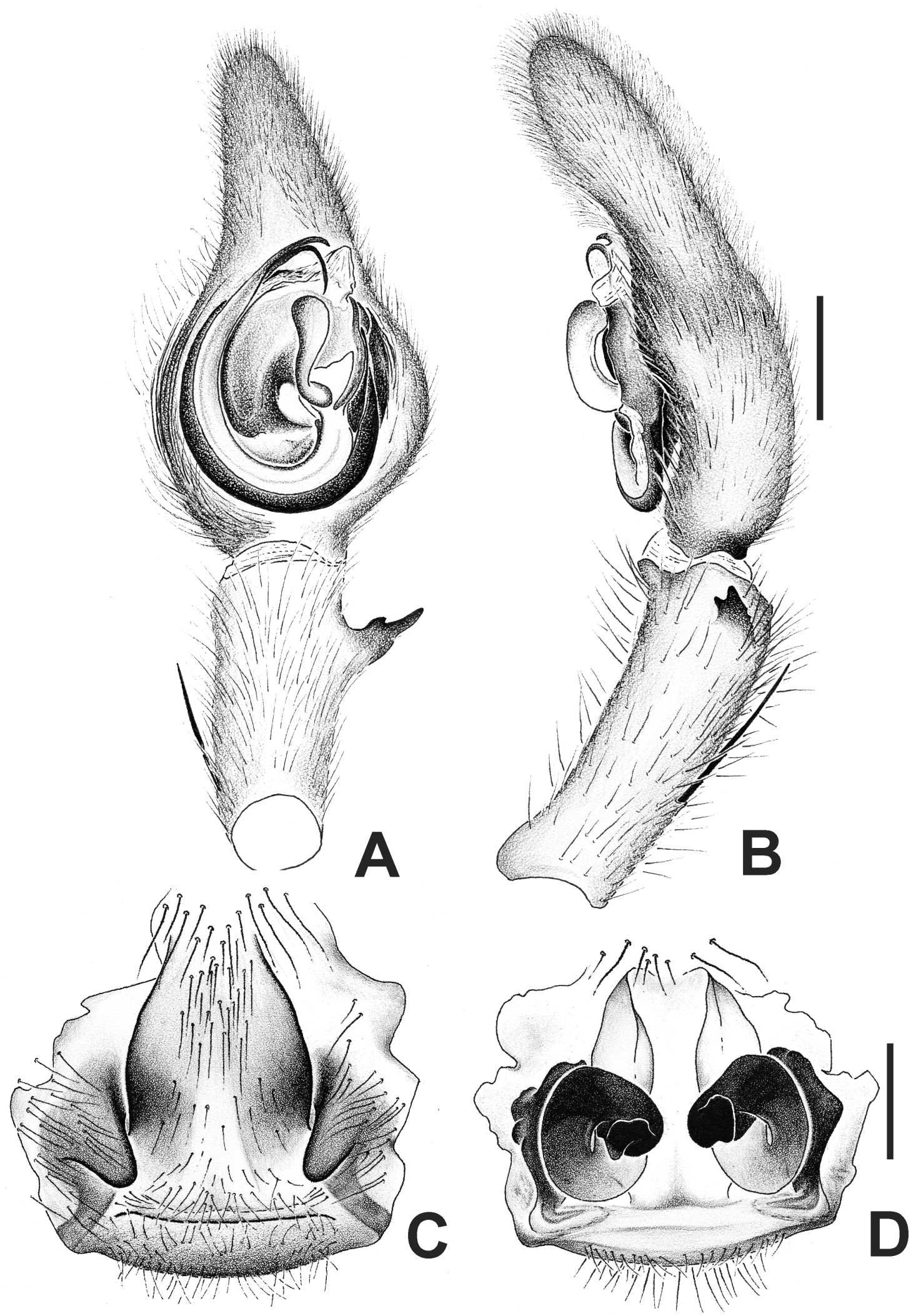

Fig. 7. Afroneutria erythrochelis (Simon, 1876) comb. nov. - A-B. Male palp. A. Ventral view. B. Retrolateral view. - C-D. Female genitalia. C. Epigynum, ventral view. D. Vulva, dorsal view. Scale bars $=1 \mathrm{~mm}$. 
POLOTOW D. \& JOCQUÉ R., A new genus of Afrotropical Ctenidae (Araneae)

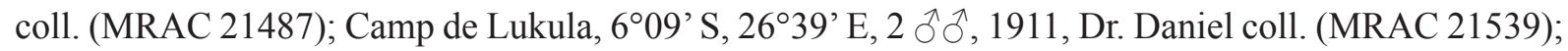
Lukula, 609' S, 2639' E, 1 ㅇ, 1953, Dr. Wauthier coll. (MRAC 74606); Kivu, Rugari, 202' S, 28 $56^{\circ}$ ' E,

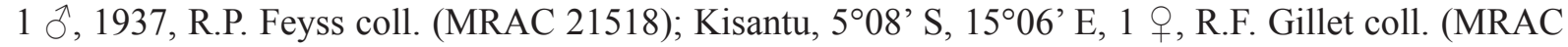

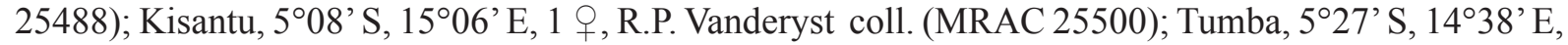

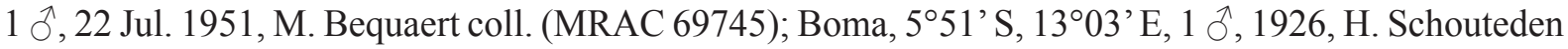
coll. (MRAC 25498); Boma, 551' S, $13^{\circ} 03^{\prime}$ E, 1 ㅇ, Nov. 1945, J. Vrydagh coll. (MRAC 25503); Bas-

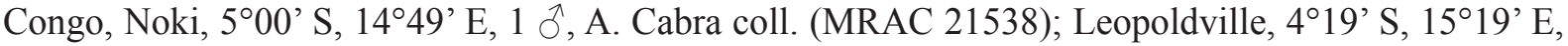

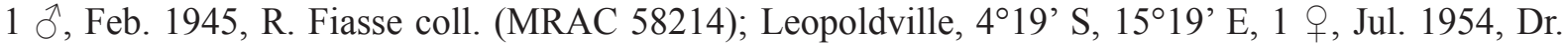

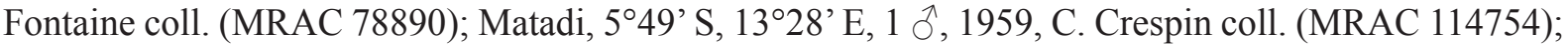

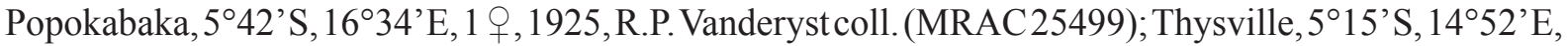

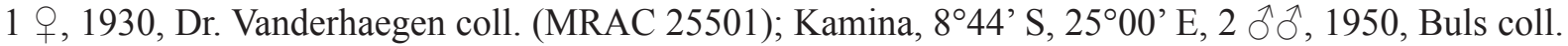

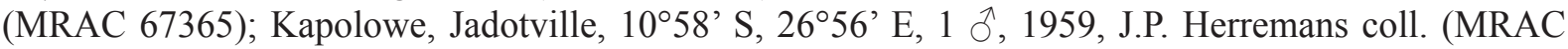
140851); Katanga, Lubumbashi, $11^{\circ} 40^{\prime}$ S, $2^{\circ} 28^{\prime}$ E, 2 우, Apr.-May 1966, J. Godeaux coll. (MRAC 131502); Elisabethville, $11^{\circ} 40^{\prime}$ S, $27^{\circ} 28^{\prime}$ E, 1 ㅇ, 1936, Richard coll. (MRAC 21503); Elisabethville,

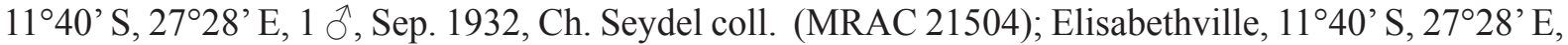

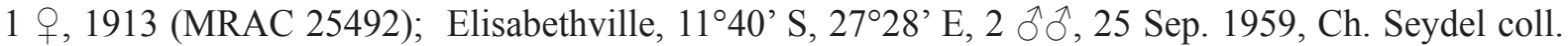

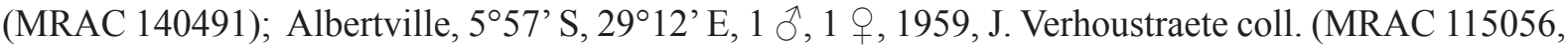

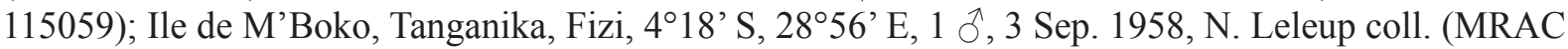

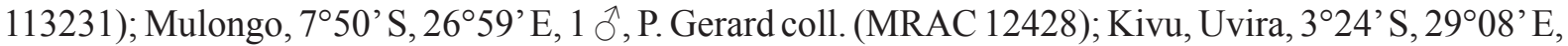
2 우, Aug. 1961, R. Kiss coll. (MRAC 120068); Kivu, Uvira, Lake Tanganika, 324’’ S, 2908’ E, 1 q,

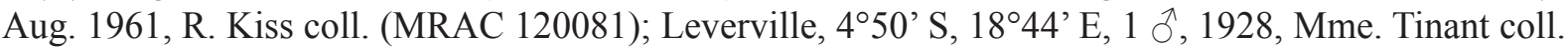

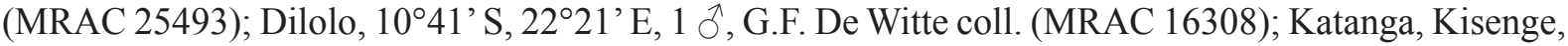
$10^{\circ} 42^{\prime}$ S, $23^{\circ} 10^{\prime}$ E, 2 우, Dec. 1964, A. Regnard coll. (MRAC 127561); Kwamouth, $3^{\circ} 11^{\prime} \mathrm{S}, 16^{\circ} 12^{\prime}$ E, 1 ㅇ, Jun. 1913, J. Maes coll. (MRAC 25494); Libenge, 339’ N, 18³8’ E, 1 ㅇ, C. Leontovitch coll. (MRAC 30274); Ndua, close to Bolobo, 2¹0' S, 16¹4' E, 1 ㅇ, Dec. 1951, N'gue coll. (MRAC 72204);

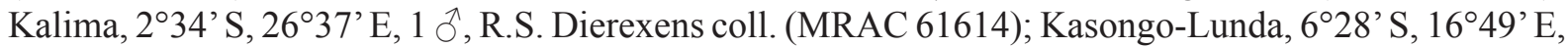

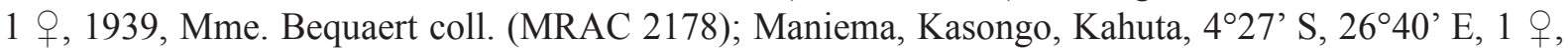
1959, P.L.G. Benoit \& Segers coll. (MRAC 140375); Bambesa, 3²6' N, 25²41' E, 1 q, Aug. 1938, J. Vrydagh coll. (MRAC 2790).

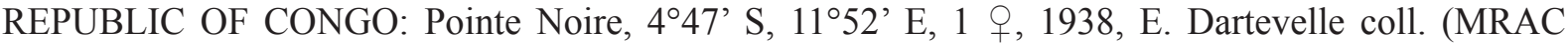
21528).

GABON: Tchibanga, 255’ S, 1059’ E, 1 , 20 Sep. 1984, A. Souche, Pauly coll. (MRAC 168750).

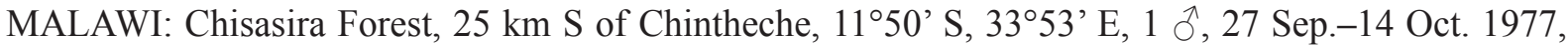

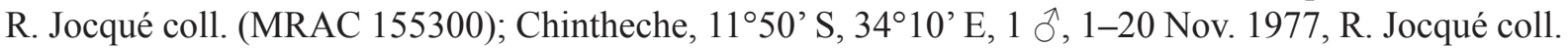
(MRAC 155304).

NAMIBIA: Andara-Kavango, Okavango River, $18^{\circ} 04^{\prime}$ S, $2^{\circ}{ }^{\circ} 29^{\prime}$ E, 1 ㅇ, Sep. 1979, M.E. Baddeley coll. (MRAC 152797).

RWANDA: Kigali, $01^{\circ} 57^{\prime}$ S, 3004’ E, 1 ㅇ, Sep. 1961, A. Lestrade coll. (MRAC 123159).

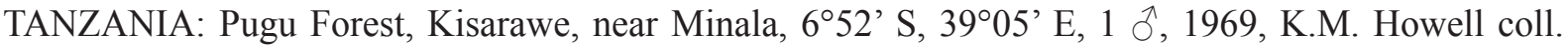
(MRAC 159272); Dar-es-Salaam, UDSM Campus, 648’ S, 39¹7’ E, 1 O’, 1970-1971, K.M. Howell coll. (MRAC 159472); Dar-es-Salaam, UDSM Campus, 6²4’ S, 39¹7’’ E, 1 ㅇ, Sep. 1980, K.M. Howell coll. (MRAC 169162).

ZIMBABWE: Bulawayo, 2009’ S, 28³6’ E, 1 q, Nov. 1964-Jan. 1965, S. Bucklin coll. (MRAC 128094). 


\section{Type material not examined}

MALAWI: Ctenus johnstoni, holotype,, , Zomba, Lake Nyassa, BMNH.

KENYA and DEMOCRATIC REPUBLIC OF THE CONGO: Ctenus carsoni, syntypes, 3 $\widehat{\partial} \widehat{\partial}$, Mombasa and Nyanza Province (Kenya) and Kavala Island, Lake Tanganyika (Democratic Republic of the Congo), Carson coll., Pasha coll., BMNH.

GHANA: Ctenus aureopubescens, holotype, §̂, probably ZMB, not found.

\section{Description}

Male (MRAC 60880)

Total length 29.10. Carapace 15.10 long and 11.45 wide. Eye diameter: AME 0.55, ALE 0.25, PME 0.72, PLE 0.66. Leg measurements: I: femur 16.30 / patella 6.80 / tibia 16.10 / metatarsus 15.90 / tarsus 4.40 / total 59.50; II: 14.30 / 6.30 / 12.40 / 14.10 / 4.20 / 51.30; III: 11.50 / 5.20 / 8.80 / 10.70 / 3.40 / 39.60; IV: $15.80 / 5.70 / 13.70 / 17.45 / 4.20 / 56.85$. Leg formula 1423. Leg spination: tibia I and II v22-2-2-2, r1-1, p1-1; III and IV v2-2-2, r1-1, p1-1; metatarsus I, II and III v2-2-2, p1-1-1, r1-1-1; IV v11-1-1-1-2, r1-1-1, p1-1-1. Legs covered by long, golden hairs (Fig. 6A-B). Palp: cymbium elongated, almost as long as tibia; conductor rectangular, curved; median apophysis drop-shaped, curved at base (Figs 7A-B, 15C-D).

\section{Female (MRAC 2178)}

Total length 32.00. Carapace 16.80 long and 12.96 wide. Eye diameter: AME 0.55, ALE 0.25, PME 0.70, PLE 0.65. Leg measurements: I: femur 15.35 / patella 7.50 / tibia 13.90 / metatarsus 13.75 / tarsus 4.40 / total 54.90; II: 14.25 / 6.60 / 11.60 / 12.20 / 3.80 / 48.45; III: 11.20 / 5.50 / 8.30 / 10.20 / 3.50 / 38.70; IV: $15.00 / 6.10 / 12.20 / 15.65 / 3.90 / 52.85$. Leg formula 1423. Leg spination: tibia I and II v2-2-2-2-2, r0, p0; III and IV v2-2-2, r1-1, p1-1; metatarsus I and II v2-2-2, r0, p0; III v2-2-2, r1-1-1, p-1-1-1; IV v1-1-1-1-1-2, r1-1-1, p1-1-1. Legs covered by long, golden hairs (Fig. 6C-D). Epigynum: triangular median field and short lateral projections of lateral fields (Fig. 7C, 12C). Dorsal spermathecae elongated, curved, ventral spermathecae short (Fig. 7D).

\section{Variation}

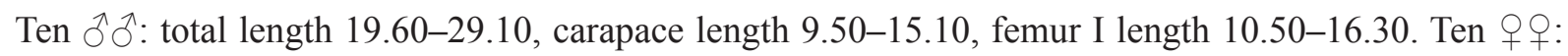
total length $31.50-33.45$, carapace length $14.50-18.25$, femur I length $12.60-16.80$.

\section{Distribution}

Angola, Burundi, Democratic Republic of the Congo, Malawi, Namibia, Republic of Congo, Rwanda, Tanzania and Zimbabwe (Fig. 16B).

\section{Afroneutria quadrimaculata sp. nov. urn:1sid:zoobank.org:act:0FF06727-624D-465E-ACA1-8A9CEFB12A50 \\ Figs $8 \mathrm{~A}-\mathrm{D}, 9 \mathrm{~A}-\mathrm{E}, 12 \mathrm{~B}, 15 \mathrm{~A}-\mathrm{B}, 16 \mathrm{~A}$}

Ctenus velox - Benoit 1977: 700, fig. 2a-d (misidentification).

\section{Diagnosis}

The males of Afroneutria quadrimaculata sp. nov. differ from those of the other species of the genus by the laminar embolus with enlarged tip and the curved, subdistal, additional projection of the embolus (Figs 9A, 15A). The females can be distinguished by the large and elongated lateral projections of the epigynum and the curved dorsal spermathecae in the vulva (Figs 9D-E, 12B). 


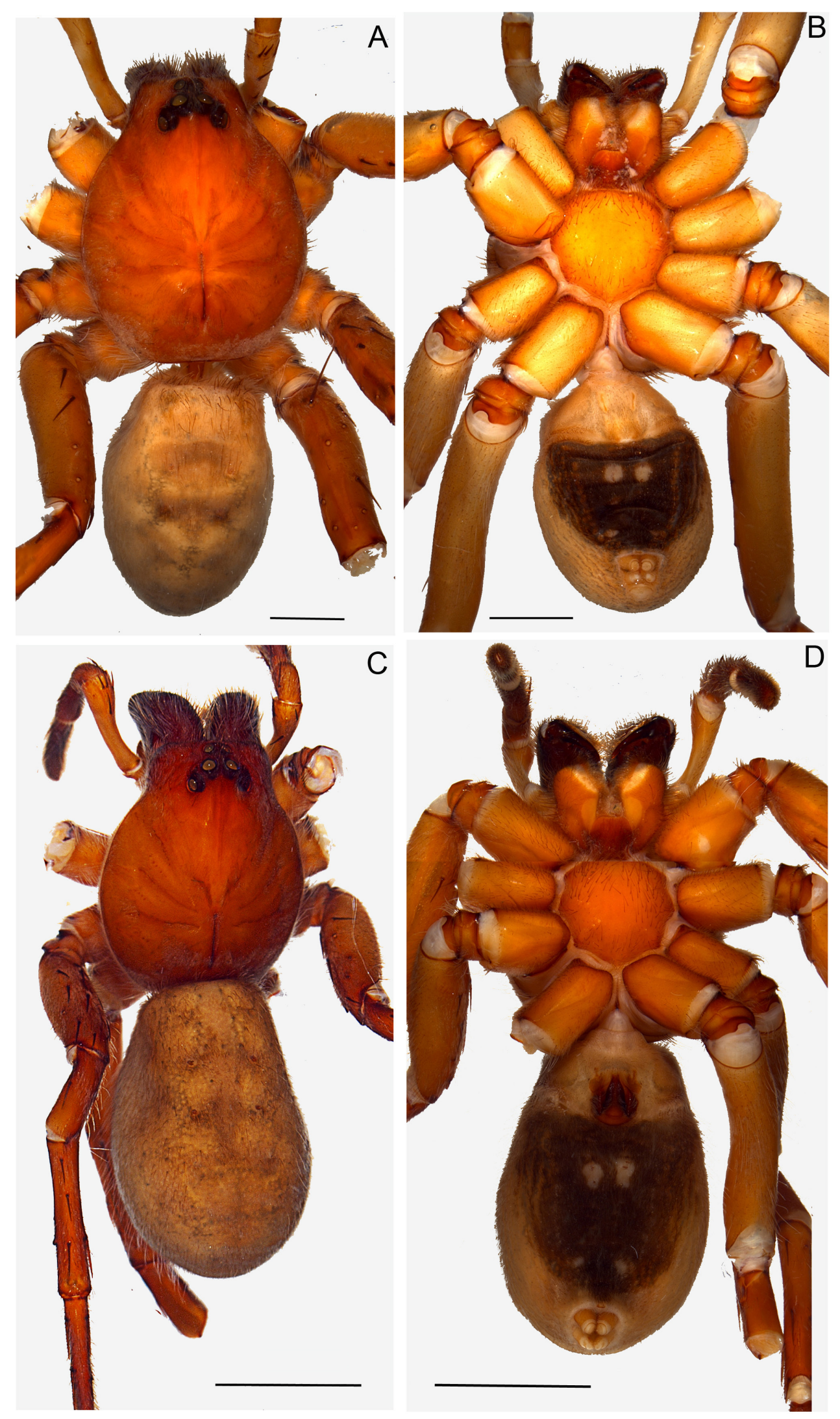

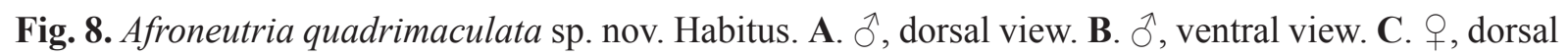
view. D. o, ventral view. Scale bars $=2 \mathrm{~mm}$. 

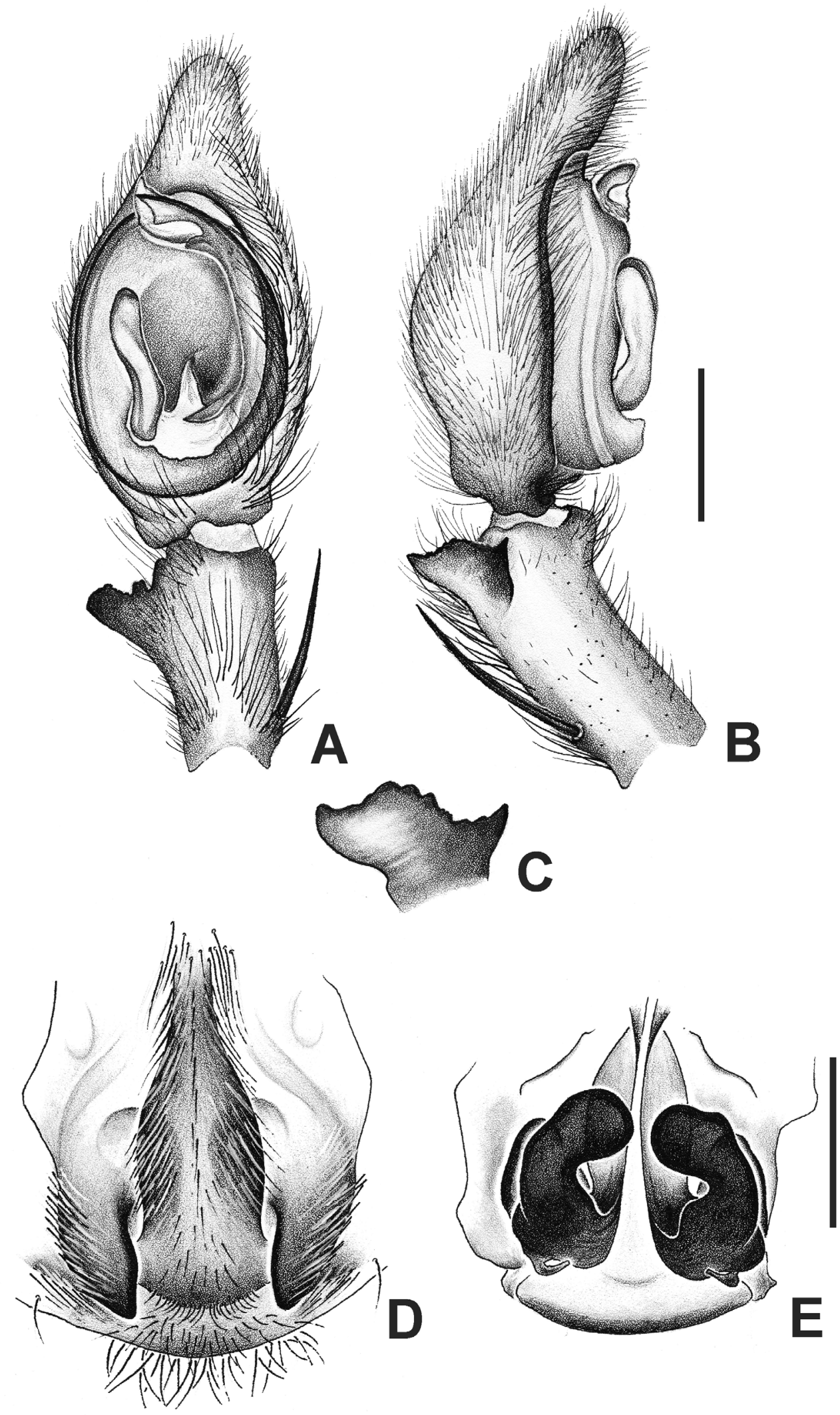

Fig. 9. Afroneutria quadrimaculata sp. nov. - A-C. Male palp. A. Ventral view. B. Retrolateral view. C. Retrolateral tibial apophysis, lateral view. - D-E. Female genitalia. D. Epigynum, ventral view. E. Vulva, dorsal view. Scale bars $=2 \mathrm{~mm}$. 
POLOTOW D. \& JOCQUÉ R., A new genus of Afrotropical Ctenidae (Araneae)

\section{Etymology}

The specific name is derived from Latin, quadri- (four) and macula- (spot), meaning to have four spots and making reference to the four white spots on the venter of the abdomen.

\section{Material examined}

\section{Holotype}

DEMOCRATIC REPUBLIC OF THE CONGO: đ̊ Jadotville, Kasompi, Katanga, Oct. 1956, Z. Bacq coll. (MRAC 90554).

\section{Paratype}

DEMOCRATIC REPUBLIC OF THE CONGO: $q$, Kisenge, Katanga, Dec. 1964, A. Regnard coll. (MRAC 127584).

\section{Additional material}

DEMOCRATIC REPUBLIC OF THE CONGO: Kasai, Bibanga, 1 , , 1940, M. Bequaert coll. (MRAC 21542); Katanga, Kisenge, 1 đ̊, 1965, Q. Regnard coll. (MRAC 128007); Katanga, Jadotville, Kasompi, 1 ㅇ, Oct. 1956 (MRAC 90555); 1 đ̊, 6 Oct. 1956 (MRAC 90596); Katanga, Luiswishi Forest, 1 q, 2 Mar. 1974, F. Malaise coll. (MRAC 149210); Katanga, L'Upemba National Park, River Lufira (680 m),

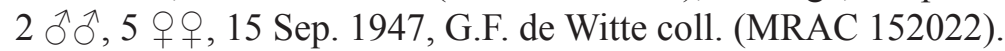

\section{Description}

\section{Male (MRAC 128007)}

Total length 14.90. Carapace 7.50 long and 5.90 wide. Eye diameter: AME 0.36, ALE 0.24, PME 0.48, PLE 0.44. Leg measurements: I: femur 9.00 / patella 3.40 / tibia 9.10 / metatarsus 8.80 / tarsus 3.30 / total 33.60; II: 8.10 / 3.30 / $7.30 / 7.80$ / 3.00 / 29.50; III: 7.00 / 2.70 / 5.50 / 6.70 / 2.50 / 24.40; IV: $9.20 / 3.10 / 8.40 / 10.90 / 3.30 / 34.90$. Leg formula 4123. Leg spination: tibia I and II v2-2-2-2-2, r1-1, p1-1; III and IV v2-2-2, r1-1, p1-1; metatarsus I, II and III v2-2-2, p1-1-1, r1-1-1; IV v1-1-1-1-1-2, r11-1, p1-1-1. Coloration: venter of abdomen black with four white spots, two behind epigastric furrow, two in front of spinnerets (Fig. 8B). Palp: cymbium slightly longer than tibia; conductor oval; median apophysis curved (Figs 9A-C, 15A-B).

\section{Female (MRAC 90555)}

Total length 20.10. Carapace 9.50 long and 7.40 wide. Eye diameter: AME 0.44, ALE 0.28, PME 0.56, PLE 0.52. Leg measurements: I: femur 8.70 / patella 4.40 / tibia 8.20 / metatarsus 7.60 / tarsus 2.70 / total 31.60; II: 7.80 / 4.10 / 6.80 / 6.70 / 2.70 / 28.10; III: 6.80 / 3.20 / 5.50 / 6.30 / 2.40 / 24.20; IV: 9.00 / $3.70 / 8.00 / 10.20 / 3.10 / 34.00$. Leg formula 4123. Leg spination: tibia I and II v2-2-2-2-2, r0, p0; III and IV v2-2-2, r1-1, p1-1; metatarsus I and II v2-2-2, r0, p0; III v2-2-2, r1-1-1, p-1-1-1; IV v1-1-1-11-2, r1-1-1, p1-1-1. Coloration: as in male (Fig. 8C-D). Epigynum: with triangular median field and elongated lateral projections of lateral fields (Figs 9D, 12B). Dorsal spermathecae long, curved, ventral spermathecae short (Fig. 9E).

\section{Variation}

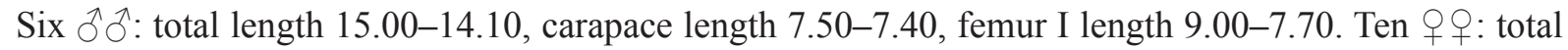
length $24.20-16.50$, carapace length $10.00-8.10$, femur I length $8.90-7.50$.

\section{Distribution}

Democratic Republic of the Congo (Fig. 16A). 
Afroneutria hybrida sp. nov.

urn:1sid:zoobank.org:act:425F304C-8F67-4DFA-A475-A3E3115CFEB8

Figs $10 \mathrm{~A}-\mathrm{B}, 11 \mathrm{~A}-\mathrm{C}, 13 \mathrm{C}-\mathrm{D}, 16 \mathrm{~B}$

\section{Diagnosis}

The males of Afroneutria hybrida sp. nov. differ from those of the other species of the genus by the laminar embolus with acute tip, the short additional subdistal projection of the embolus and the truncated RTA (Figs 11A-B, 13C-D).

\section{Etymology}

The specific name is derived from Latin ibrida, meaning a mongrel, specifically the offspring of a tame sow and a wild boar, and making reference to the similarity of the morphology to that of Afroneutria velox comb. nov. and A. quadrimaculata sp. nov.

\section{Material examined}

\section{Holotype}

TANZANIA: §̊̄, Kambai Forest Reserve, Muheza District, Tanga Region, 459’ S, 3841’ E, ZMUC.
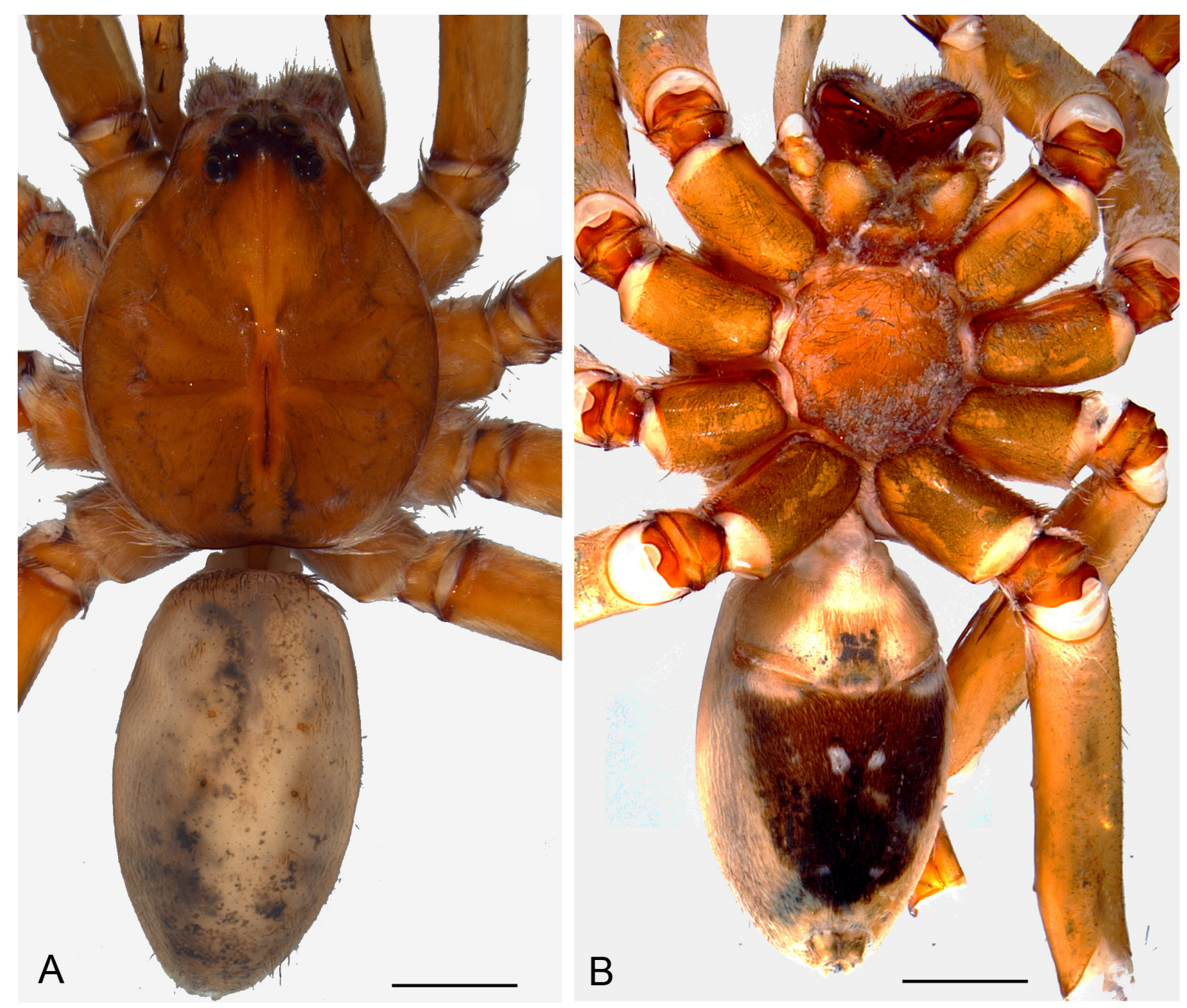

Fig. 10. Afroneutria hybrida sp. nov. Habitus, $\widehat{\jmath}$. A. Dorsal view. B. Ventral view. Scale bars $=2 \mathrm{~mm}$. 


\section{Paratype}

TANZANIA: $1 \hat{\jmath}$, same collection data as holotype, ZMUC.

\section{Additional material}

None.

\section{Description (holotype)}

Total length 12.40. Carapace 6.50 long and 5.50 wide. Eye diameter: AME 0.32, ALE 0.19, PME 0.60, PLE 0.56. Leg measurements: I: femur 9.70 / patella 3.20 / tibia 10.60 / metatarsus 11.40 / tarsus 4.70 / total 39.60; II: 8.70 / 3.10 / 8.90 / 9.50 / 3.50 / 33.70; III: 7.50 / 2.40 / 6.70 / 7.70 / 3.10 / 27.40; IV: 10.20 / $2.60 / 10.00 / 13.20 / 4.30 / 40.30$. Leg formula 4123. Leg spination: tibia I and II v2-2-2-2-2, r1-1, p11; III and IV v2-2-2, r1-1, p1-1; metatarsus I, II and III v2-2-2, p1-1-1, r1-1-1; IV v1-1-1-1-1-2, r1-1-1, p1-1-1. Coloration: venter of abdomen black, with two white spots placed transversely behind epigastric
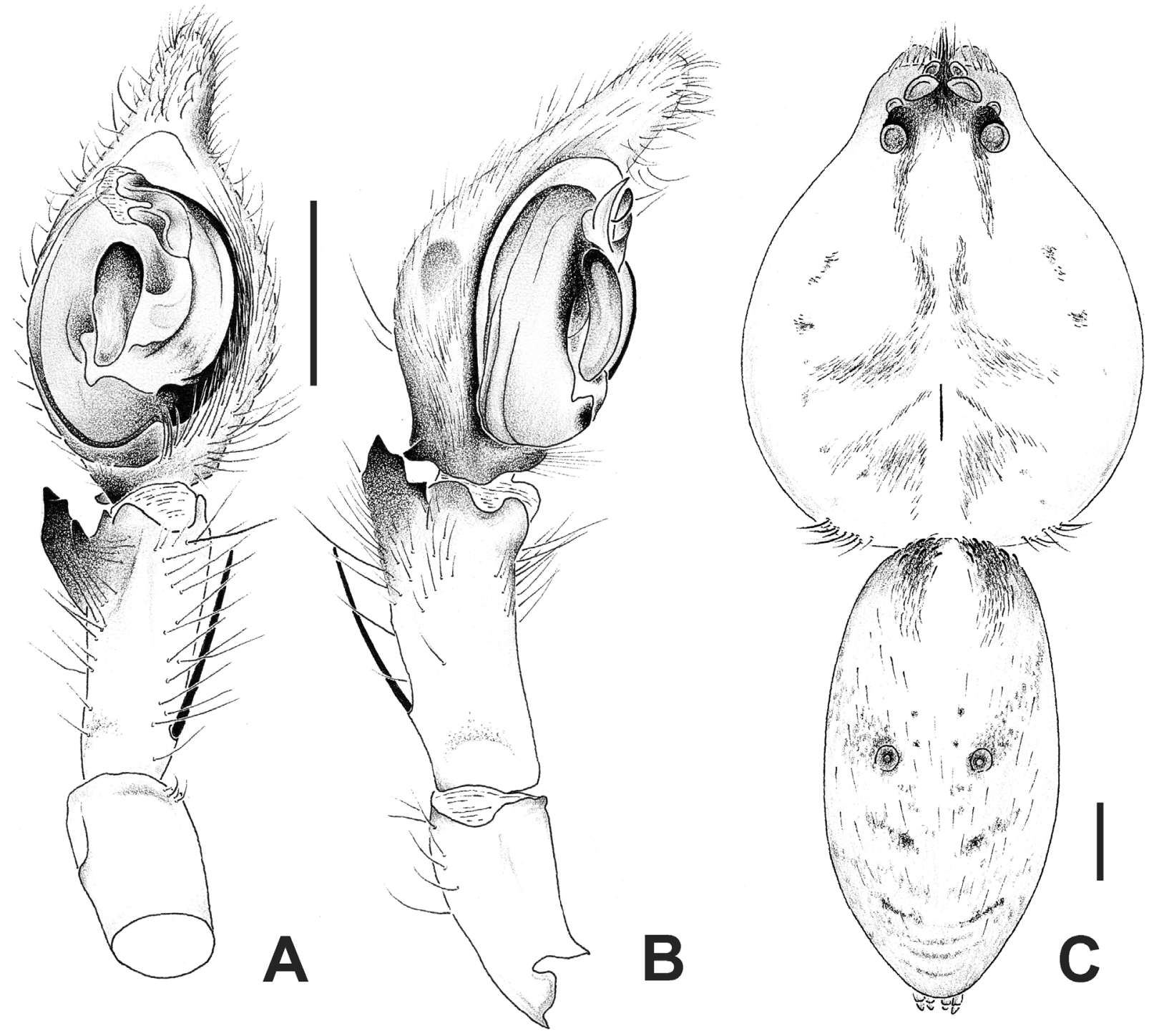

Fig. 11. Afroneutria hybrida sp. nov., $\widehat{\partial}$. A. Palp, ventral view. B. Palp, retrolateral view. C. Habitus, dorsal view. Scale bars $=1 \mathrm{~mm}$. 
furrow (Fig. 10B). Palp: cymbium elongated, longer than tibia; conductor oval; median apophysis straight (Figs 11A-B, 13C-D).

\section{Distribution}

Tanzania (Fig. 16B).
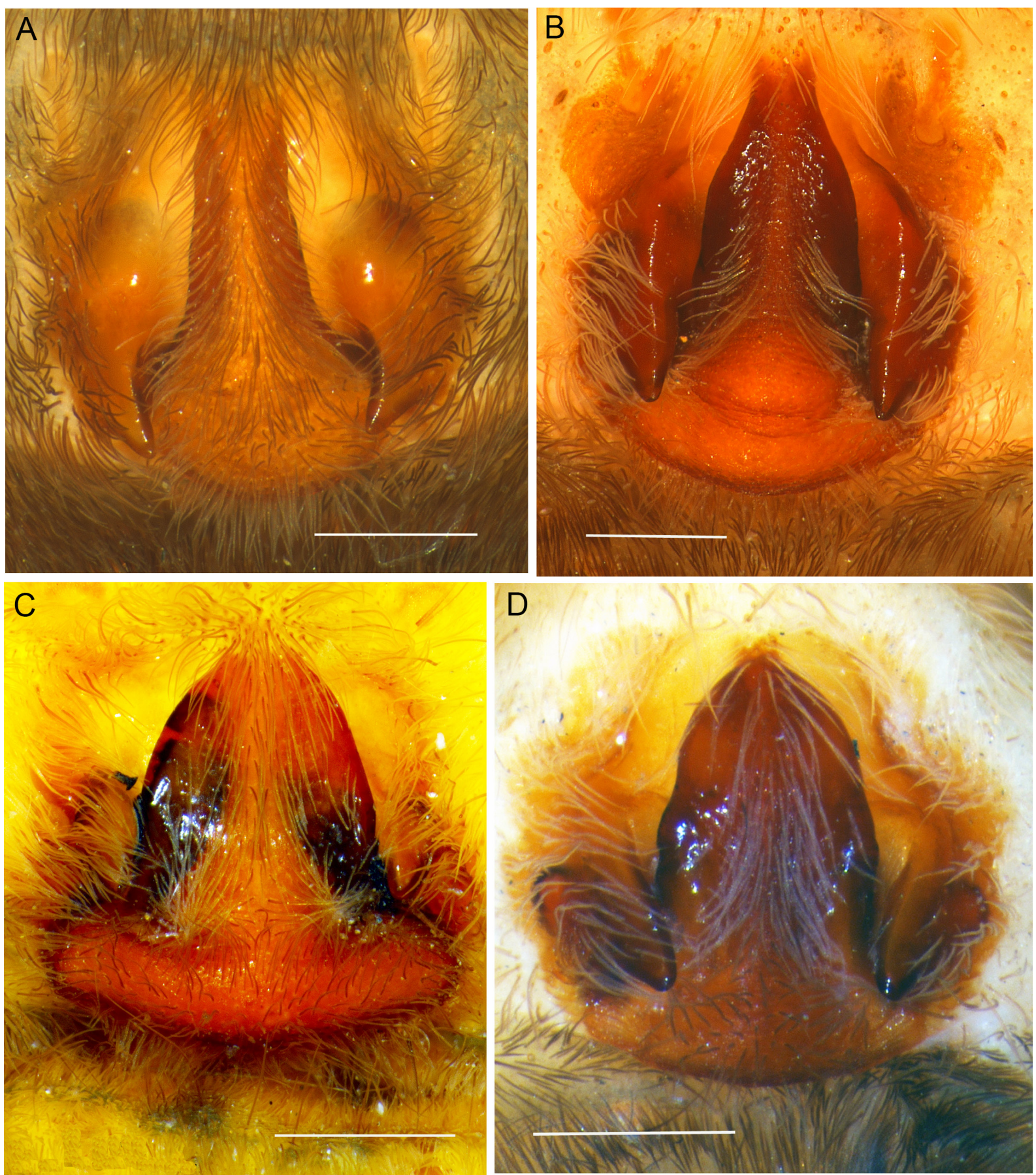

Fig. 12. Epigynum, ventral view. A. Afroneutria immortalis (Arts, 1912) comb. nov. B. Afroneutria quadrimaculata sp. nov. C. Afroneutria erythrochelis (Simon, 1876) comb. nov. D. Afroneutria velox (Blackwall, 1865) comb. nov. Scale bars $=1 \mathrm{~mm}$. 


\section{Discussion}

Afroneutria gen. nov. is proposed based on the results of the cladistic analysis presented in Polotow \& Brescovit (2014). In that analysis, three of the species treated here were used: Afroneutria erythrochelis comb. nov., A. immortalis comb. nov. and A. velox comb. nov. The clade appeared as sister-group of a clade formed by Ctenus sinuatipes F.O. Pickard-Cambridge, 1897 and C. curvipes (Keyserling, 1881), both from Central America (Polotow \& Brescovit 2014: fig. 3). The Afroneutria clade is supported by a unique elongated projection in the apical area of the embolus (Polotow \& Brescovit 2014: character 25), also present in the species described here, A. quadrimaculata sp. nov. and $A$. hybrida sp. nov.

The Afrotropical region houses 123 species of Ctenidae, distributed in eight genera: Africactenus Hyatt, 1954, Anahita Karsch, 1879, Apolania Simon, 1898, Caloctenus Keyserling, 1877, Ctenus Walckenaer, 1805, Petaloctenus Jocqué \& Steyn, 1997, Thoriosa Simon, 1910 and Trogloctenus Lessert, 1935 (Platnick 2015). Viridasius Simon, 1889 and Vulsor Simon, 1889 are excluded from the count, because they belong to another family according to Polotow et al. (in press). Ctenus contains the largest number of species (more than 70). The recent redescription of the Neotropical type species, Ctenus dubius, by Brescovit \& Simó (2007) and cladistic analyses (Silva 2003; Polotow \& Brescovit 2009) indicated that the genus is polyphyletic as currently delimited. Thus, the description of Afroneutria gen. nov. is helping to redefine Ctenidae and the polyphyletic Ctenus in the Afrotropical region.
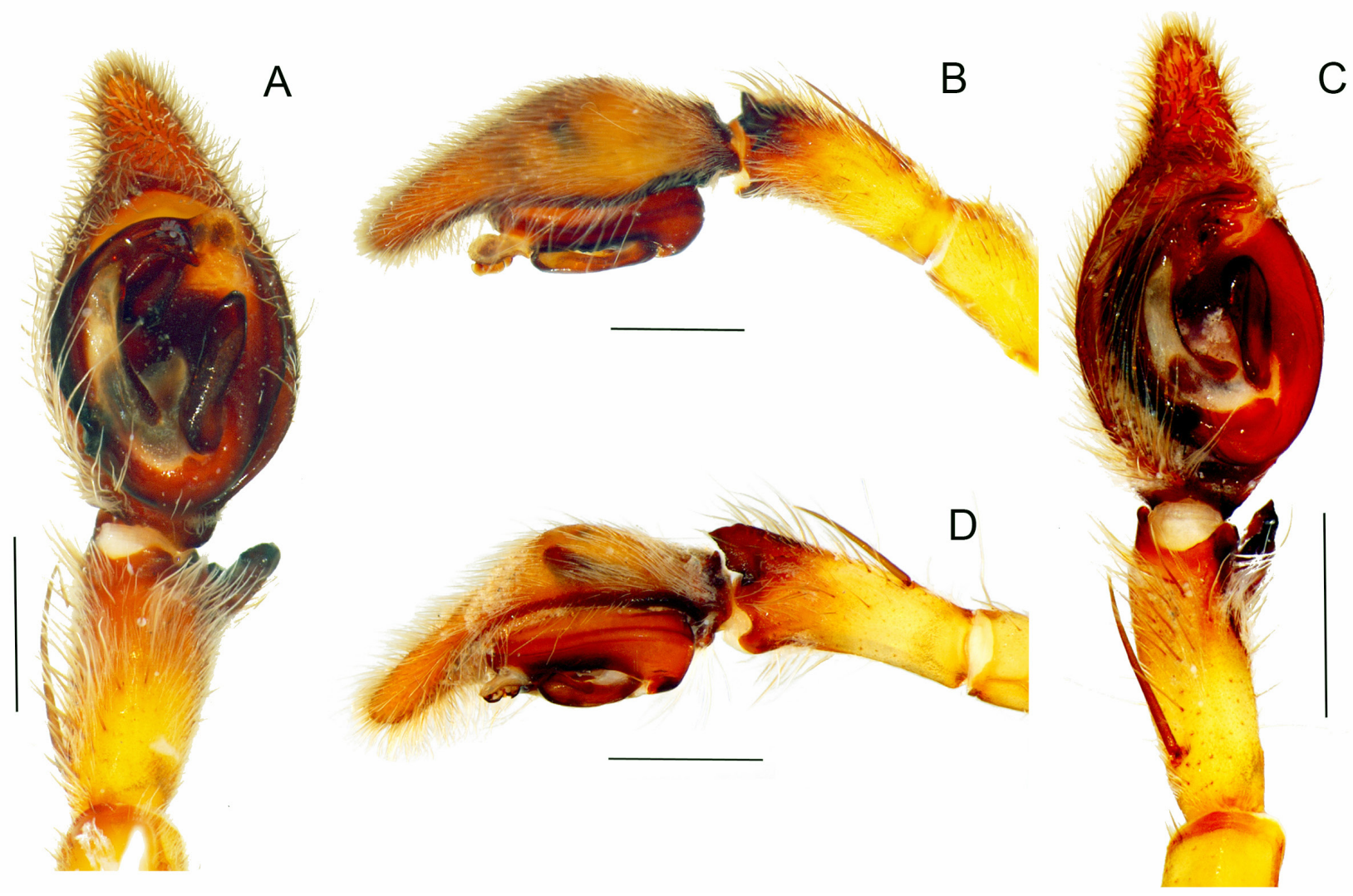

Fig. 13. Male palp. - A-B. Afroneutria velox (Blackwall, 1865) comb. nov. A. Ventral view. B. Retrolateral view. - C-D. Afroneutria hybrida sp. nov. C. Ventral view. D. Retrolateral view. Scale bars $=1 \mathrm{~mm}$. 

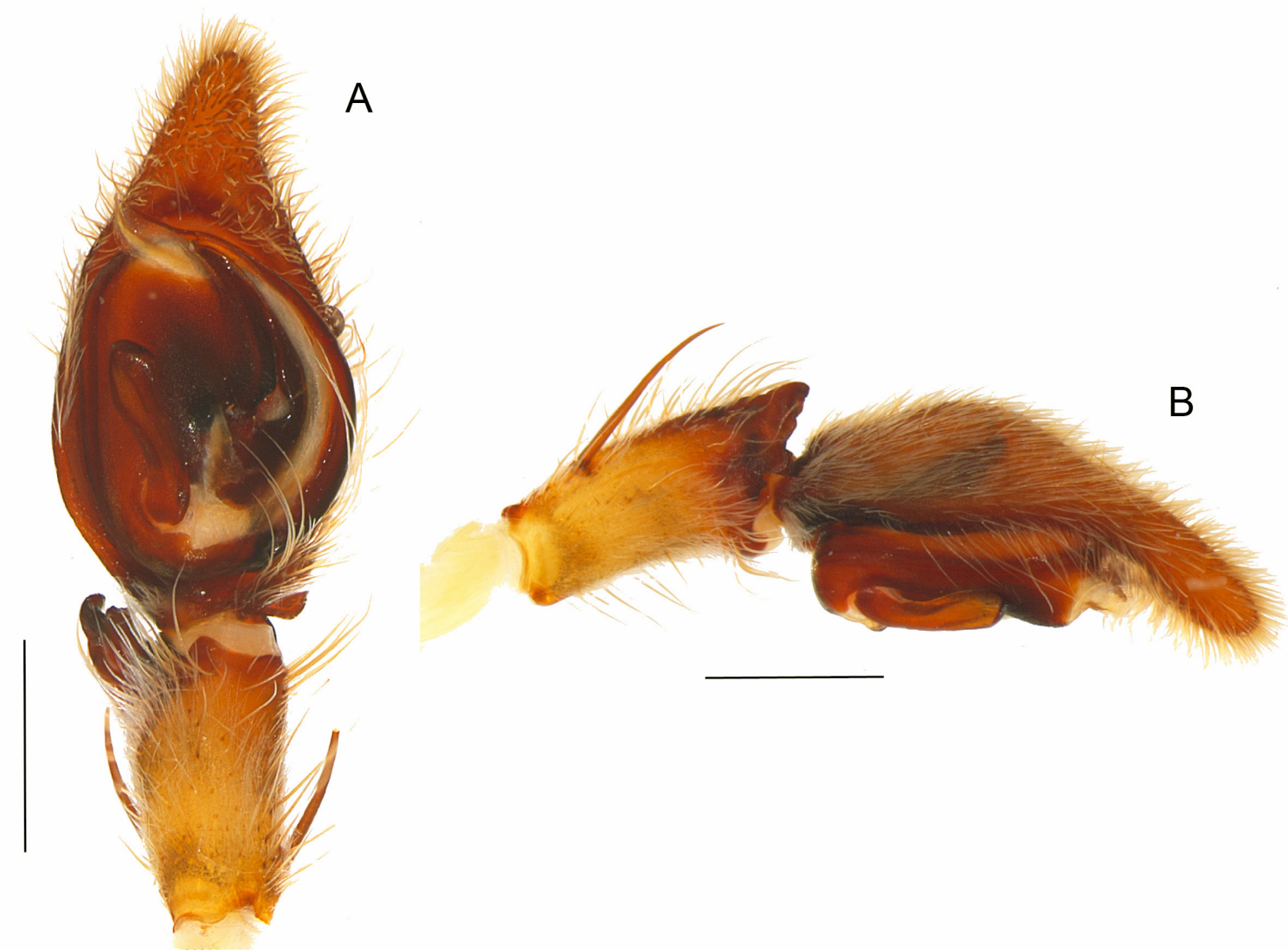

Fig. 14. Afroneutria immortalis (Arts, 1912) comb. nov. Male palp. A. Ventral view. B. Retrolateral view. Scale bars $=1 \mathrm{~mm}$.
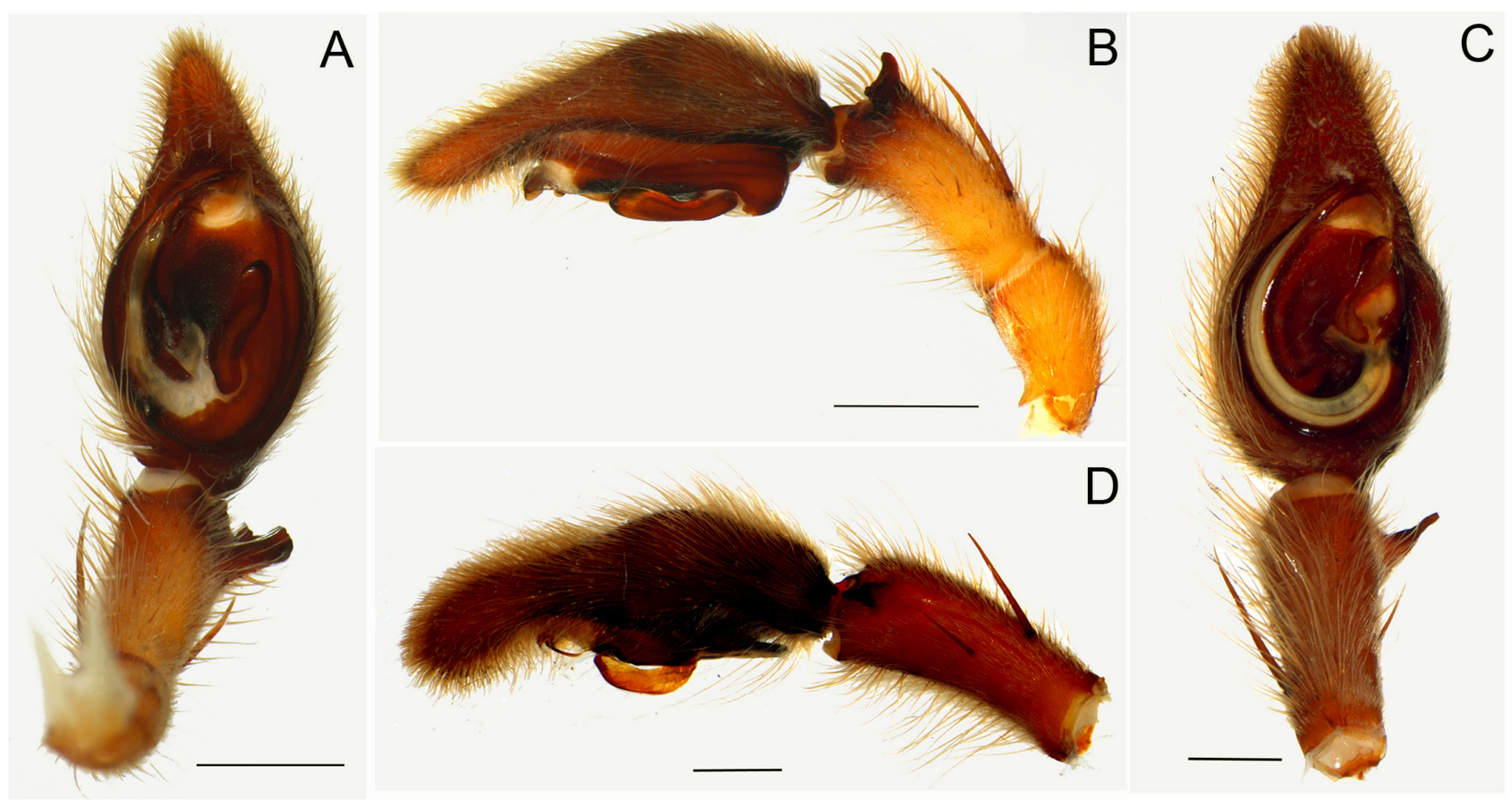

Fig. 15. Male palp. - A-B. Afroneutria quadrimaculata sp. nov. A. Ventral view. B. Retrolateral view. - C-D. Afroneutria erythrochelis (Simon, 1876) comb. nov. C. Ventral view. D. Retrolateral view. Scale bars $=1 \mathrm{~mm}$. 

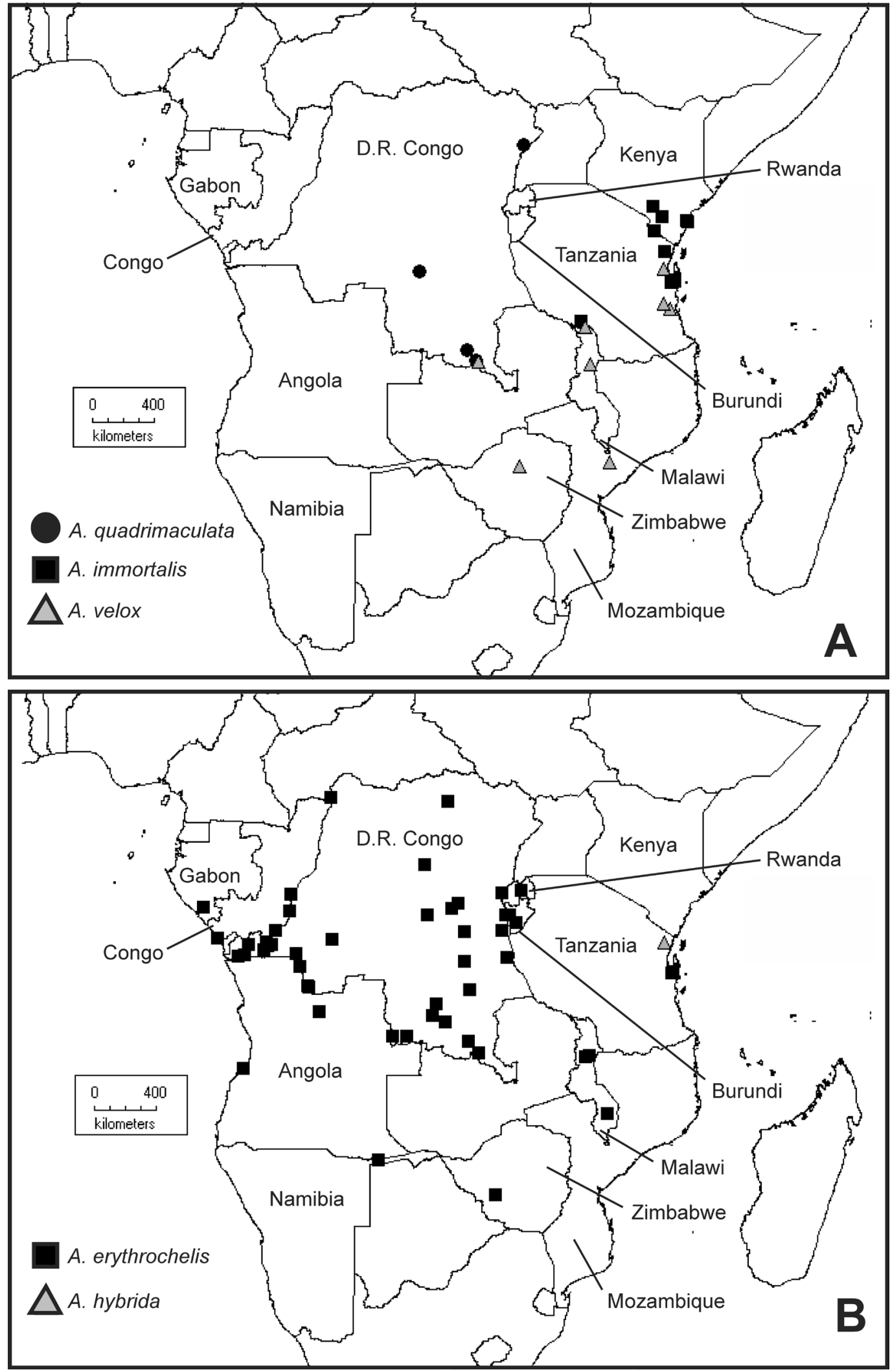

Fig. 16. Distribution records of species of Afroneutria gen. nov. on the African continent. A. Distribution of A. velox (Blackwall, 1865) comb. nov., A. immortalis (Arts, 1912) comb. nov. and A. quadrimaculata sp. nov. B. Distribution of $A$. erythrochelis (Simon, 1876) comb. nov. and A. hybrida sp. nov. 


\section{Acknowledgments}

Financial support for this study was provided by the Fundação de Amparo a Pesquisa do Estado de São Paulo - FAPESP (06/55230-7, doctorate fellowship) and the Belgian National Focal Point to the Global Taxonomy Initiative at the Royal Museum for Central Africa, Tervuren. We would like to thank all curators who kindly lent critical specimens for this research.

\section{References}

Arts L. des. 1912. Zusammenstellung der afrikanischen Arten der Gattung Ctenus. Jahrbuch der Hamburgischen Wissenschaftlichen Anstalten 29 (2): 183-218. http://www.biodiversitylibrary.org/ item/94918\#page/201/mode/1up [accessed 19 Mar. 2015]

Benoit P.L.G. 1977. Etudes sur les Ctenidae africains (Araneae) V. Gen. Ctenus Walck. - groupe erythrochelis. Revue zoologique africaine 91: 698-703.

Blackwall J. 1865. Descriptions of recently discovered species and characters of a new genus of Araneidea from the East of Central Africa. The Annals and Magazine of Natural History, $3^{\text {rd }}$ Series, 16: $336-352$.

Bösenberg W. \& Lenz H. 1895. Ostafrikanishe Spinnen gesammelt von Herrn Dr. F. Stuhlmann in den Jahren 1888 und 1889. Jahrbuch der Hamburgischen Wissenschaftlichen Anstalten 12 (2): 27-51. http:// www.biodiversitylibrary.org/item/101782\#page/397/mode/1up [accessed 19 Mar. 2015]

Brescovit A.D. \& Simó M. 2007. On the Brazilian Atlantic Forest species of the spider genus Ctenus Walckenaer, with the description of a neotype for C. dubius Walckenaer (Araneae, Ctenidae, Cteninae). Bulletin of the British Arachnological Society 14: 1-17. http://dx.doi.org/10.13156/arac.2007.14.1.1

Hyatt K.H. 1954. The African spiders of the family Ctenidae in the collections of the British Museum (Natural History). The Annals and Magazine of Natural History, 12 ${ }^{\text {th }}$ Series, 7: 877-894. http://dx.doi. org/10.1080/00222935408651809

Karsch F. 1879. West-afrikanische Arachniden, gesammelt von Herrn Stabsarzt Dr. Falkenstein. Zeitschrift für die gesammten Naturwissenschaften 52: 329-373.

Lessert R. de. 1915. Arachnides de l'Ouganda et de l'Afrique orientale allemande (voyage du Dr J. Carl dans la region des lacs de l'Afrique centrale). Revue suisse de zoologie 23: 1-80. http://www. biodiversitylibrary.org/item/40642\#page/11/mode/1up [accessed 19 Mar. 2015]

Pickard-Cambridge F.O. 1898. On the cteniform spiders of Africa, Arabia and Syria. Proceedings of the Zoological Society of London 1898: 13-32. http://www.biodiversitylibrary.org/item/97095\#page/47/ mode/1up [accessed 19 Mar. 2015]

Polotow D. \& Brescovit A.D. 2009. Revision and cladistic analysis of Isoctenus and description of a new neotropical genus (Araneae, Ctenidae, Cteninae). Zoological Journal of the Linnean Society 155: 583-614. http://dx.doi.org/10.1111/j.1096-3642.2008.00452.x

Polotow D. \& Brescovit A.D. 2014. Phylogenetic analysis of the tropical wolf spider subfamily Cteninae (Arachnida, Araneae, Ctenidae). Zoological Journal of the Linnean Society 170: 333-361. http://dx.doi. org/10.1111/zoj.12101

Polotow D., Carmichael A. \& Griswold C.E. In press. Total evidence analysis of the phylogenetic relationship of Lycosoidea spiders (Araneae, Entelegynae). Invertebrate Systematics.

Ramírez M.J. 2014. The morphology and phylogeny of dionychan spiders (Araneae, Araneomorphae). Bulletin of the American Museum of Natural History 390: 1-374. http://dx.doi.org/10.5531/sd.sp.5 
Silva D. 2003. Higher-level relationships of the spider family Ctenidae (Araneae: Ctenoidea). Bulletin of the American Museum of Natural History 274: 1-86. http://dx.doi.org/10.1206/00030090(2003)274<0001:HLROTS $>2.0$. CO;2

Simó M. \& Brescovit A.D. 2001. Revision and cladistic analysis of the Neotropical spider genus Phoneutria Perty, 1833 (Araneae, Ctenidae), with notes on related Cteninae. Bulletin of the British Arachnological Society 12 (2): 67-82.

Simon E. 1876. Étude sur le arachnides du Congo. Bulletin de la Société zoologique de France 1: 215-224. http://www.biodiversitylibrary.org/item/110342\#page/22/mode/1up [accessed 19 Mar. 2015]

Simon E. 1897. Histoire Naturelle des Araignées. Librairie encyclopédique de Roret, Paris. http://dx.doi. org/10.5962/bhl.title.51973 [accessed 19 Mar. 2015]

Strand E. 1906. Tropisch-afrikanische Spinnen des Kgl. Naturalien-kabinetts in Stuttgart. Jahreshefte des Vereins für Vaterländische Naturkunde in Württemberg 62: 13-103. http://www.biodiversitylibrary. org/item/33679\#page/131/mode/1up [accessed 19 Mar. 2015]

Strand E. 1907. Afrikanische Spinnen (exkl. Aviculariiden), hauptsächlich aus dem Kapland. Zoologische Jahrbücher, Abteilung für Systematik 25: 557-731. http://www.biodiversitylibrary.org/ item/38161\#page/569/mode/1up [accessed 19 Mar. 2015]

World Spider Catalog. 2014. World Spider Catalog, version 15.5. Natural History Museum Bern [online]. Available from http://wsc.nmbe.ch [accessed 19 Mar. 2015]

Manuscript received: 22 December 2014

Manuscript accepted: 2 March 2015

Published on: 28 April 2015

Topic editor: Koen Martens

Desk editor: Danny Eibye-Jacobsen

Printed versions of all papers are also deposited in the libraries of the institutes that are members of the EJT consortium: Muséum national d'Histoire naturelle, Paris, France; Botanic Garden Meise, Belgium; Royal Museum for Central Africa, Tervuren, Belgium; Natural History Museum, London, United Kingdom; Royal Belgian Institute of Natural Sciences, Brussels, Belgium; Natural History Museum of Denmark, Copenhagen, Denmark. 